\title{
Greater Manchester State of the Voluntary, Community and Social Enterprise Sector 2017
}

\section{$A$ report on social and economic impact}

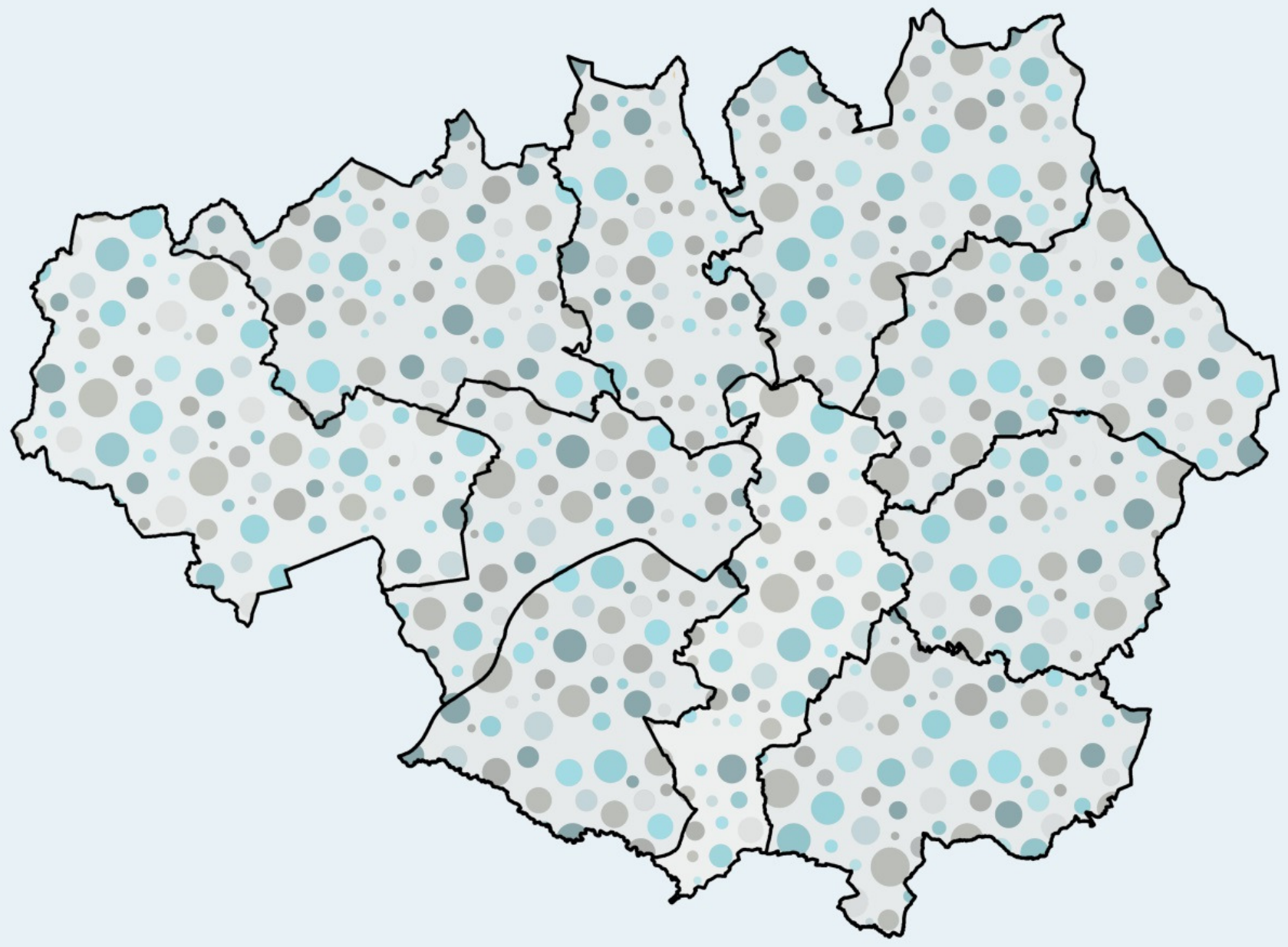

Sheffield Hallam

Centre for

Regional Economic

University and Social Research 


\section{Greater Manchester State of the Voluntary, Community and Social Enterprise Sector 2017}

Centre for Regional Economic and Social Research

Sheffield Hallam University

Chris Damm

loannis Prinos

Elizabeth Sanderson

DOI: $10.7190 /$ cresr.2017.8245386329

June 2017 


\section{Acknowledgements}

This research has been commissioned by GMCVO, 10GM ${ }^{1}$ and Stockport Council and undertaken by the Centre for Regional Economic and Social Research (CRESR) at Sheffield Hallam University. This Greater Manchester report forms part of a wider collection of reports: there is one for each of the individual local authority areas participating in the study ${ }^{2}$. The full collection of reports can be downloaded from the CRESR website (http://www.shu.ac.uk/research/cresr/reports) and the websites of the project partners.

In completing the report we are particularly grateful to members of the Research Steering Group ${ }^{3}$ and the staff teams at each project partner for their support in developing and administering the survey and for their input into earlier drafts of the report. We are also grateful to the many employees and volunteers from across the voluntary, community and social enterprise sector who took the time to complete a questionnaire or participate in a focus group.

\section{Contact information}

For CRESR:

Name: Elizabeth Sanderson Research Fellow

Address: Unit 10 Science Park

City Campus

Howard Street

Sheffield

S1 1WB

Tel: $\quad 01142254814$

Email: e.sanderson@shu.ac.uk
For GMCVO:

Name: Alex Whinnom

Chief Executive

Address: St Thomas Centre

Ardwick Green North

Manchester

M12 6FZ

Tel: $\quad 01612771000 / 1004$

Email: alex.whinnom@gmcvo.org.uk www.gmcvo.org.uk

\footnotetext{
${ }^{1} 10 \mathrm{GM}$ is a joint venture by the Greater Manchester Voluntary Sector Infrastructure Organisations (Action Together in Oldham and Tameside, Bolton CVS, CVS Rochdale, Macc, Salford CVS, VCAT, Wigan \& Leigh CVS)

2 The areas are: Bolton, Manchester, Oldham, Rochdale, Salford, Stockport, Tameside and Wigan.

${ }^{3}$ The following organisations were represented on the Research Steering Group: CRESR, Salford CVS (lead partner), Greater Manchester Centre for Voluntary Organisation (GMCVO), Bolton CVS, Macc, Action Together in Oldham and Tameside and CVS Rochdale.
} 


\section{Definitions}

This report is about the 'state of the voluntary, community and social enterprise sector in Greater Manchester'. At various times the voluntary sector has been known as the 'voluntary and community sector' or the 'third sector' whilst the current Government talks a lot about 'civil society'. In this report, when we talk about the voluntary sector in Greater Manchester, we mean voluntary organisations, community groups, the community work of faith groups, and those social enterprises where there is a wider accountability to the public via a board of trustees or a membership and all profits will be reinvested in their social purpose. 


\section{Foreword}

This report offers a timely update on the state of the voluntary, community and social enterprise (VCSE) sector, at the moment Greater Manchester elects its first city region mayor and embarks on a fourth ambitious devolution deal.

Three rounds of public spending cuts imposed by central government since 2011, correlated with deprivation, have hit Greater Manchester hard. They have occurred alongside increasing amounts of devolution from national government departments to the new Greater Manchester Combined Authority, Health and Social Care Partnership and now the GM Mayor. The combined impact of these huge and sometimes conflicting trends is hard to unpick, but it is clear that Greater Manchester is a rapidly changing place, and increasingly unlike anywhere else in the UK. All organisations operating within the city region are affected in some way by these enormous changes.

Reduced public funds and a long recession have had a severe impact on the welfare and wellbeing of local people, and the VCSE sector, along with the faith sector, is the refuge of last resort for increasing numbers. We are seeing rising poverty and inequalities, with neither public nor VCSE sector able to meet the increasing levels of need and demand. To make matters worse, the incidence of poverty, poor health, low skills, poor housing and poor opportunities correlates largely with existing structural inequalities experienced by disadvantaged groups such as BME communities, disabled people etc. thus increasing them further The Brexit vote in Greater Manchester approximately reflected affluence versus deprivation, and one immediate noticeable after-effect of the referendum was a spike in hate crime and intolerance towards BME, LGBTQ and other minority groups.

We note with concern that work is no longer a passport out of poverty - increasing numbers of people are operating in a grey economy in which they may work long hours for effectively much less than the minimum wage, and still cannot afford to support their families. The number of foodbanks in Greater Manchester, run by VCSE and faith organisations, has risen over seven years from two to 50 plus - with most users being working families.

Despite all the key agencies, including GMCVO, signing a pledge in 2006 to reduce health inequalities across GM - this has not yet occurred. Both life expectancy and healthy life expectancy remain unacceptably low compared with national averages, and the incidence of many life-constraining conditions such as mental illness and cancer unacceptably high. It remains to be seen whether health and social care devolution will help; we must hope it will.

Despite very considerable goodwill within public sector institutions, in the context of such enormous funding cuts, there is inevitably reduced investment in VCSE development, support and commissioning in most areas, and specific disinvestment in "infrastructure" in some. This has put a great many individual organisations under strain, and they have been responding in different ways - by adapting their business models, or reducing activity and going out of business. There is heavy pressure on around 500 organisations that have grown strong on public commissioning, and are now very squeezed by both high cuts and high demand. We have already seen for example the loss of our law centres, most of community transport sector and most volunteering infrastructure. On the other hand there has been some asset transfer, of buildings and spaces, and some 
successful examples of communities taking on the running of public facilities such as parks and libraries.

The impact of public funding cuts within Greater Manchester is not even. Some authorities have been hit much harder than others, and these are the same authorities which did not benefit over the years from national investments i.e. Bury, Trafford and Stockport. These are at the most challenging end of the spectrum and are most obliged to think radically, which they have done in different ways.

It is interesting and very positive that VCSE infrastructure including GMCVO has never been so well-placed and in demand with public sector colleagues - albeit never so poorly resourced! The relationship between the public and VCSE sectors seems to be mutating from a somewhat paternalistic, funding-led approach to a much more equal partnership which could lead to a stronger alliance with each working to its strengths. Meanwhile many more links are being built between VCSE and private sector organisations (especially SMEs, but also with bigger ethical businesses) universities and housing organisations. Much very exciting multi-sectoral work is being tested, often with good results.

We observe anecdotally on the ground a phenomenon which is also reflected in the research that there is rapid change and turnover within the VCSE sector itself in response to current pressures, challenges and new opportunities. Some organisations are losing capacity to adapt and to take action to secure their own futures. They are facing more competition for what used to be "VCSE funding sources" from national charities, housing organisations, universities and out-ofarea organisations, and apparently there are reduced numbers and quality of applications for Big Lottery funding. On the other hand there is definitely a rise in the numbers of CICs, social enterprises and similar initiatives involved in primary trading or wealth creation and/or making use of a purely volunteer workforce, and with no expectation of public money. There are a number of growing social enterprise sectors - and anecdotally, a surprising proportion of these seem to be led by people from BME backgrounds and by young people, which is again very encouraging.

In summary, it's a mixed picture. But the research does overall indicate that although there are considerable pressures on the VCSE sector and on individual organisations, and it remains potentially fragile, on the whole the sector continues as a large, diverse and active movement with considerable social and economic impact across the city region. There is also good evidence that many organisations are adapting and developing to meet new needs and ensure their own sustainability. There is much to be hopeful about.

Alex Whinnom, Chief Executive, GMCVO 


\section{Contents}

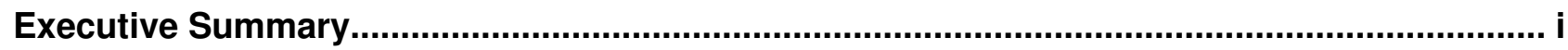

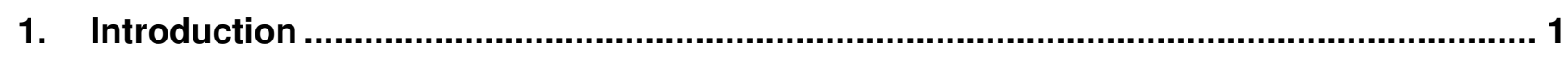

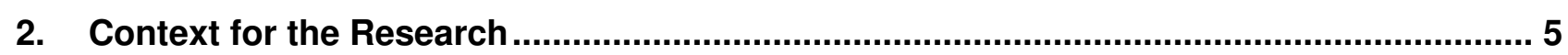

3. What the Voluntary Community and Social Enterprise Sector in Greater Manchester

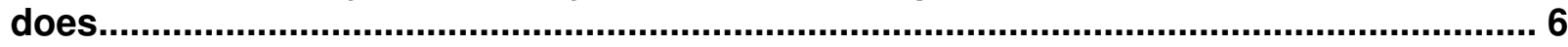

4. Who the Voluntary Community and Social Enterprise Sector in Greater Manchester

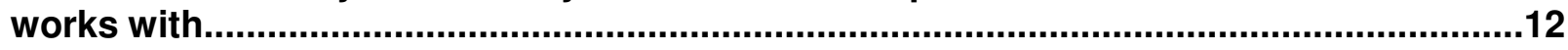

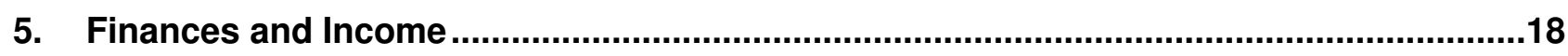

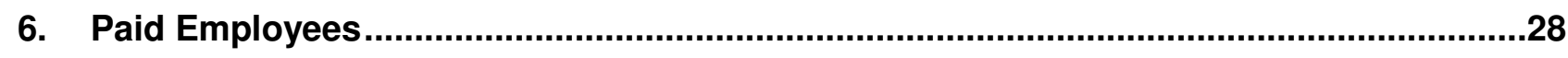

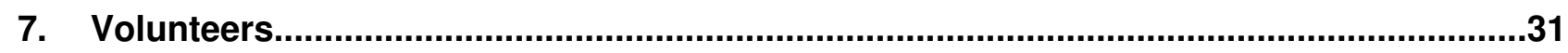

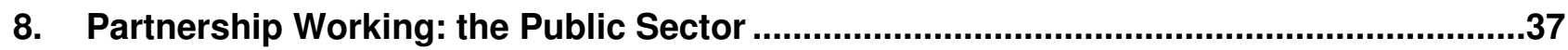

9. Partnership Working: the Private Sector ......................................................................44

10. Partnership Working: Voluntary Community and Social Enterprise Organisations........47

11. The Future

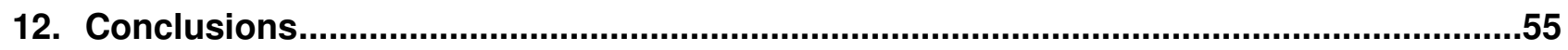

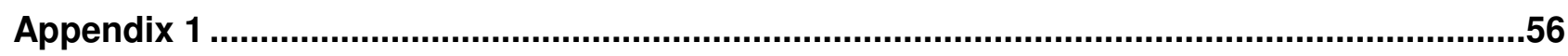




\section{Executive Summary}

This report provides the main findings of research aimed at improving the understanding of the social and economic impact of the voluntary, community and social enterprise (VCSE) sector in Greater Manchester. The key objective of the research was to provide a comprehensive overview of the sector in Greater Manchester at the start of 2017 and to show some of the ways it has changed since the previous survey of 2013.

In this summary we answer eleven key questions about the sector and its role across Greater Manchester.

\section{Q1. How many organisations are there?}

There are an estimated 15,890 organisations working in the VCSE sector in Greater Manchester and the vast majority of organisations are micro or small (90 per cent less than $£ 100,000)$ :

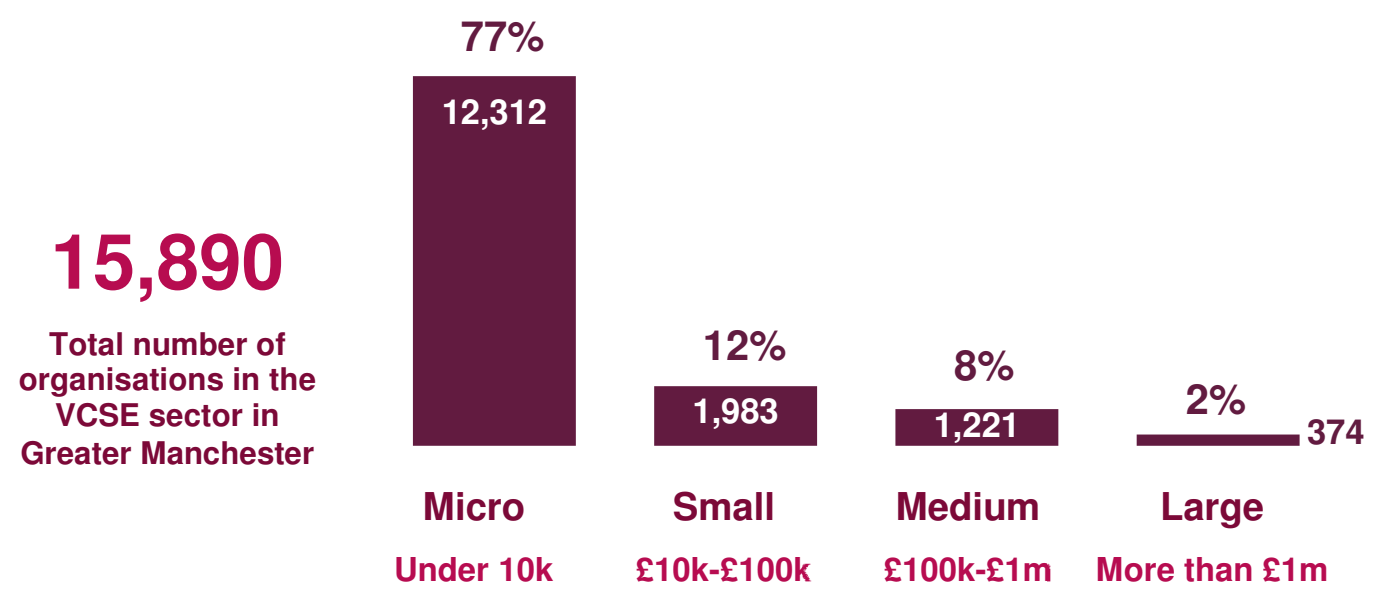

\section{Q2. Who benefits from their work?}

The client groups served by the largest proportions of organisations can be broadly characterised as being demographic. One third of organisations surveyed identified 'everyone' as their main clients, users or beneficiaries.

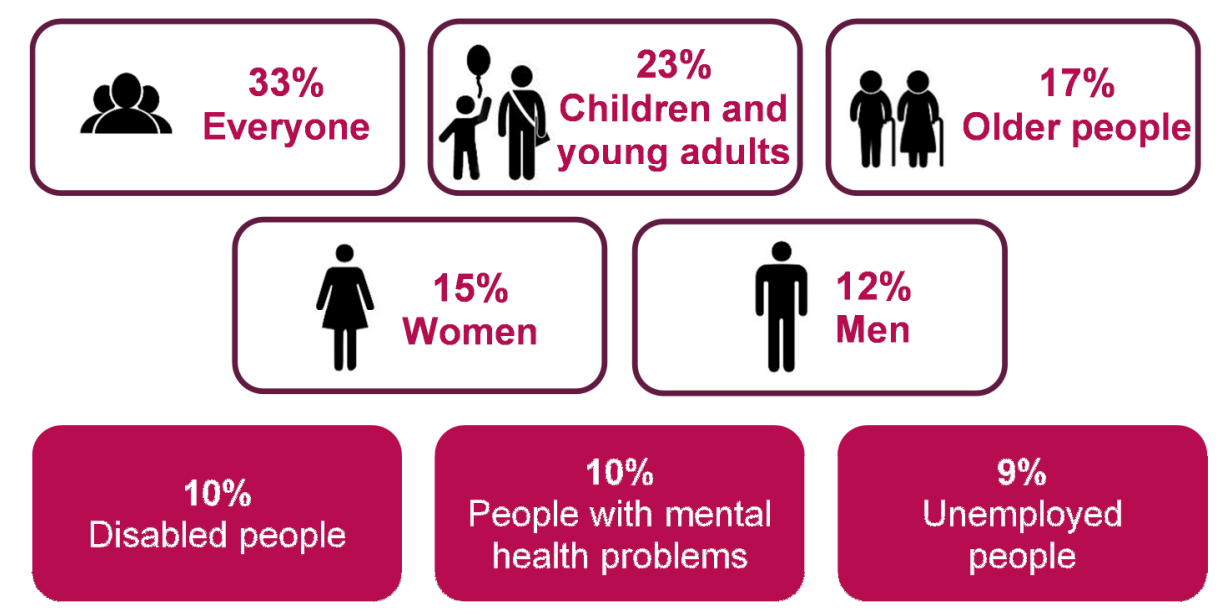


It is estimated that the VCSE sector in Greater Manchester made:

\section{9 million interventions with clients, users or beneficiaries in the past year}

The VCSE sector works at a range of different geographical levels: both across and beyond Greater Manchester; the local authority area, and specific communities and neighbourhoods within it, are the main focus for a majority of organisations:

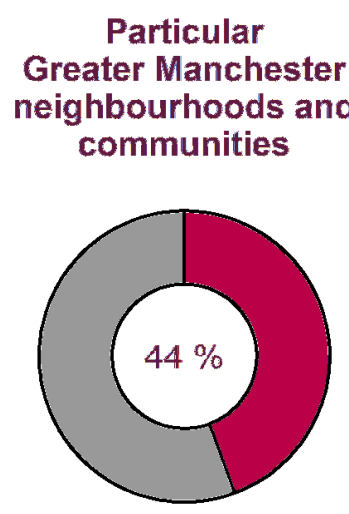
Across one Greater Manchester Local Authority area

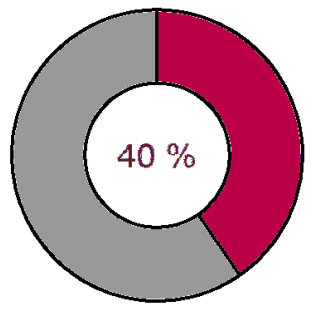

\section{Q3. What does the VCSE sector in Greater Manchester do?}

The areas with the greatest proportion of organisations working in them are:
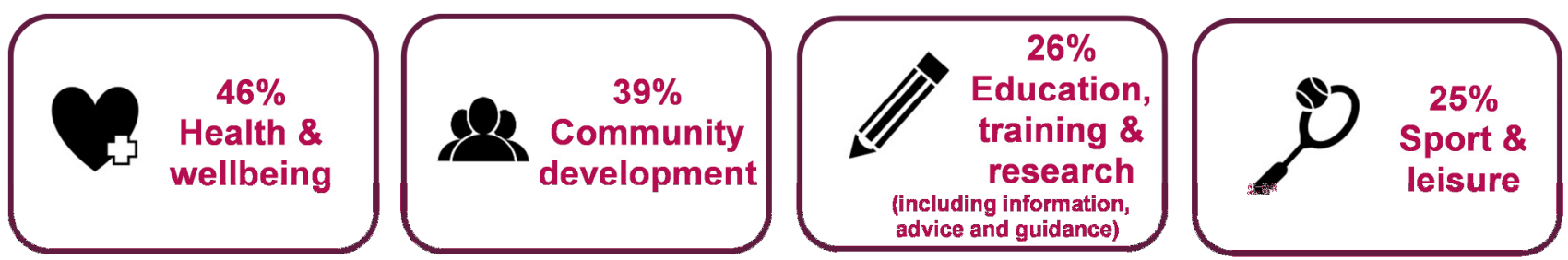

MAIN AREAS IN 2012/13:

HEALTH AND WELLBEING - 37\% COMMUNITY DEVELOPMENT - 37\%

\section{Q4. What is the income of the VCSE sector in Greater Manchester?}

Total income in $2014 / 15$ is estimated to be $\mathbf{£} 1.321 \mathbf{b n}$, an increase of three per cent compared to 2013/14.

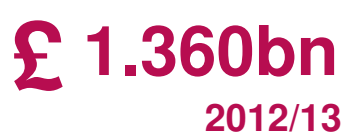

Micro and small organisations account for nine out of ten organisations in the VCSE sector in Greater Manchester but less than ten per cent of total income.

\section{$\%$ change}

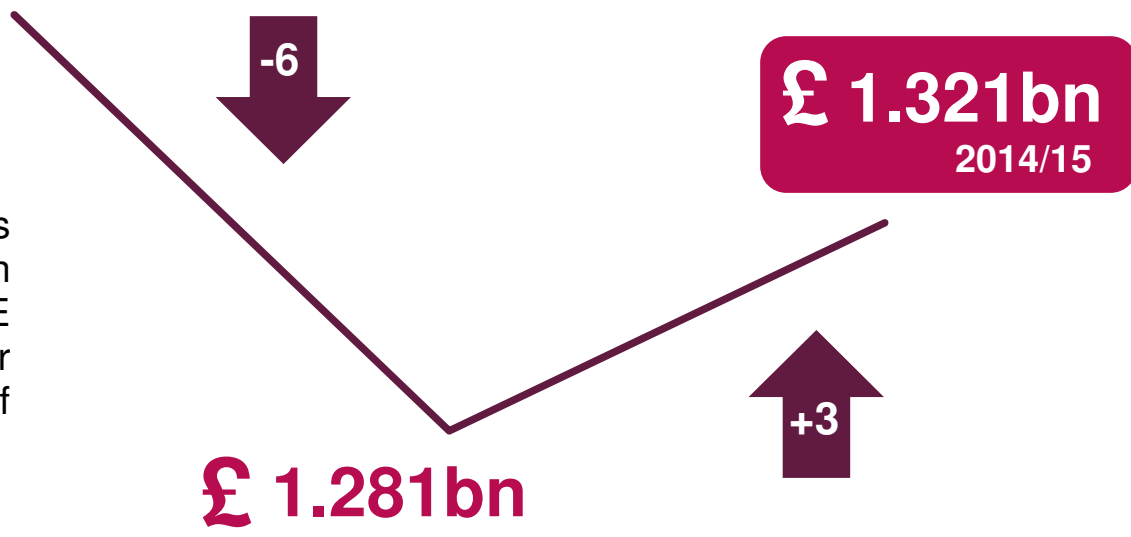

\section{3/14}

Across Greater Manchester micro and small organisations experienced year on year reductions in total income between 2012/13 and 2014/15.

By contrast medium and large organisations saw a reduction in total income between $2012 / 13$ and $2013 / 14$ but then an increase between 2013/14 and 2014/15. But income is still below 2012/13 levels. 


\section{Q5. Where does the VCSE sector receive its funding from?}

\section{$84 \%$ have at least one source of non-public sector funds \\ $76 \%$ IN 2012/13}

INCLUDING:

Fundraising (received by 50 per cent of respondents)

Grants from charitable trusts and foundations (42 per cent)

Charging for goods and services (31 per cent)

MAIN SOURCES OF FUNDING IN 2012/13:

FUNDRAISING - 54\%

GRANTS FROM CHARITABLE TRUSTS \&

FOUNDATIONS - 39\%

MEMBERSHIPS FEES \& SUBSCRIPTIONS- $37 \%$

\section{$68 \%$ have at least one source of public sector funds \\ $53 \%$ IN 2012/13}

INCLUDING:

Local authorities (received by 40 per cent of respondents)

Grants administered by local infrastructure organisations (17 per cent)

Local public sector health bodies (15 per cent)

MAIN SOURCES OF FUNDING IN 2012/13:

LOCAL AUTHORITIES - 71\%

LOCAL PUBLIC SECTOR HEALTH BODIES - 15\%

GRANTS ADMINISTERED BY LOCAL

INFRASTRUCTURE ORGANISATIONS - 9\%

\section{Q6. How sustainable is the VCSE sector in Greater Manchester?}

\section{The survey highlights some areas for concern:}

- 50 per cent of respondents reported increasing their expenditure but only $\mathbf{4 0}$ per cent had experienced an increase in income

- 28 per cent of respondents reported a decrease in income but only 16 per cent reduced their expenditure

- 33 per cent reported a reduction in their financial reserves compared to 26 per cent reporting an increase.

Total annual income

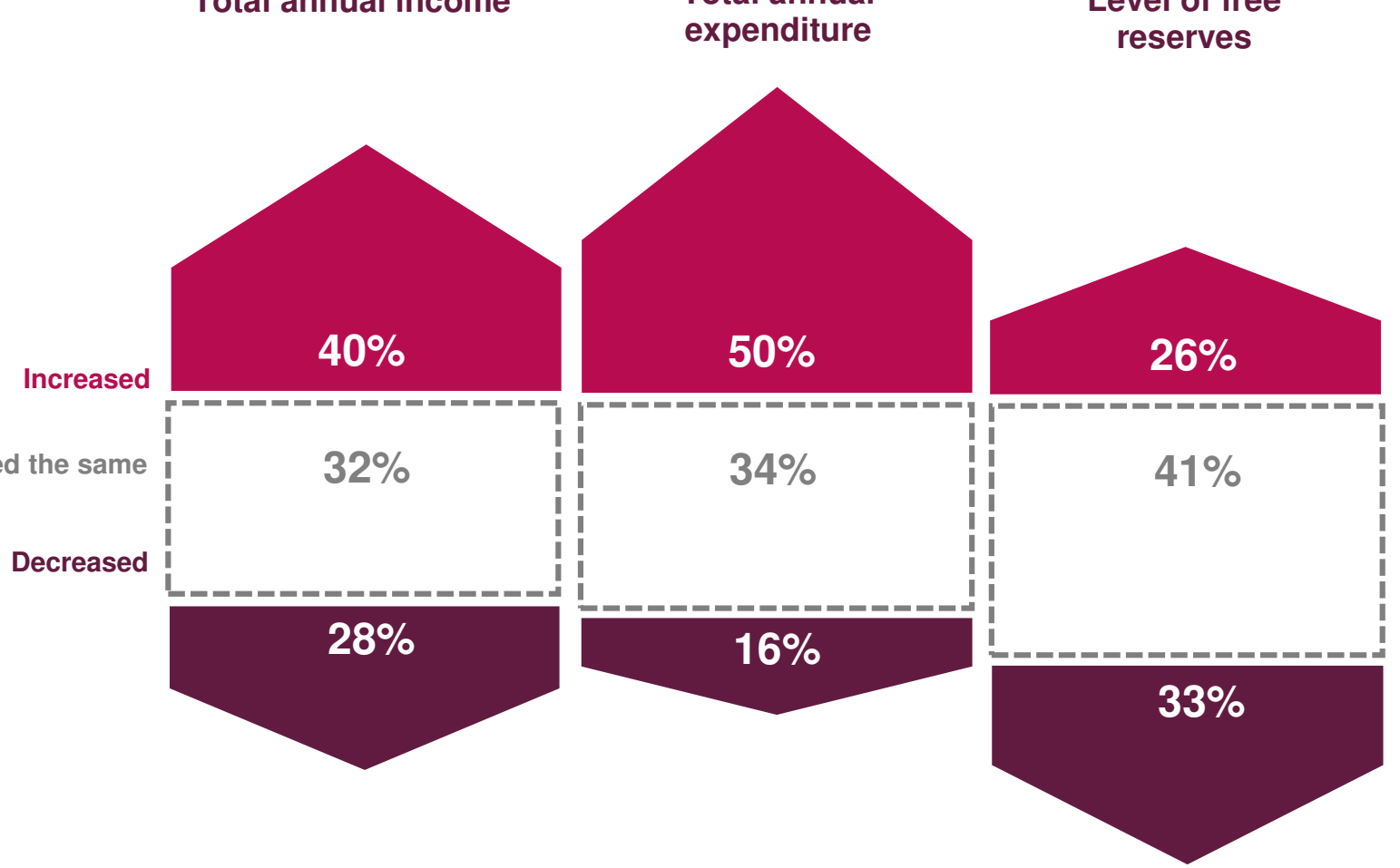


The precarious financial situation of some organisations is further emphasised by the state of their reserves:

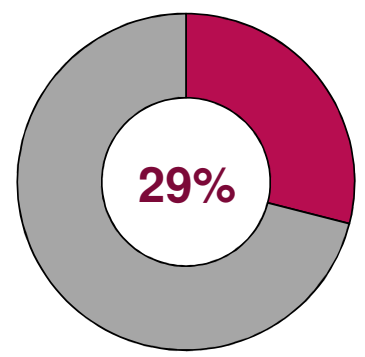

Proportion of organisations with reserves less than one month's expenditure

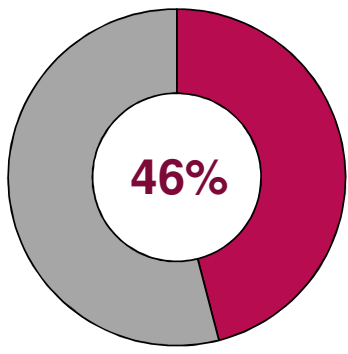

Proportion of organisations with reserves less than

25 per cent of annual expenditure

\section{Q7. Who works and volunteers in the VCSE sector?}

The sector is supported by ${ }^{4}$ :

\section{1,800 volunteers $\&$ committeel board members $(355,800$ volunteers and 105,900 committee/board members)}

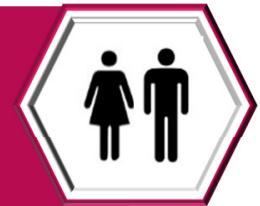

who donated

1.1 milllion hours of their time per week

$£ 973$ million per annum = estimated economic contribution of volunteers

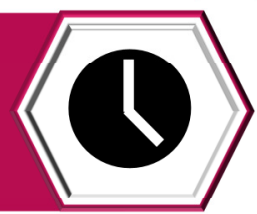

The VCSE sector is also a significant employer. There are an estimated:

\section{8,600 FTE paid staff employed in the VCSE sector in Greater Manchester}

\section{3 million per annum
contributed to the economy by paid staff of Greater
Manchester vCSE sector organisations}

\footnotetext{
${ }^{4}$ The approach to valuing the contribution of volunteers used by this study is to value the output that they produce. In effect this is the value to society of the goods and services that volunteers produce. This can be estimated by multiplying the number of FTE volunteers by the estimated gross value added (GVA) per FTE employee. This study used Greater Manchester GVA per employee averaged across the following two VCSE sectors: education and human health and social work activities. This approach is more consistent with the approach suggested by New Economy, an economic development body whose purpose is to create economic growth and prosperity for Greater Manchester. However a difference remains with the approach suggested by New Economy. Whereas the current study has used average GVA per FTE employee to value the GVA of paid staff the New Economy approach uses staff costs and multiplies this by the ratio of 'compensation of employees' to GVA: a multiplying factor of 1.5. However, staff cost data was not collected as part of this study so the New Economy method could not be fully adopted.
} 


\section{Q8. How good are relationships with public sector bodies?}

$84 \%$ had some dealings with at least one local authority: 16 per cent had a great amount of dealings and 36 per cent had a fair amount of dealings.

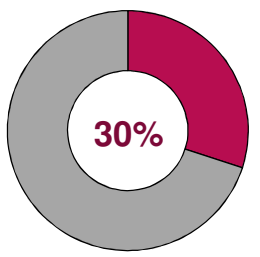

... of VCSE organisations are satisfied with their ability to influence local authorities, up slightly from 28 per cent in 2012/13; but more respondents (36 per cent) are satisfied with their ability to influence their most frequent other public sector contact

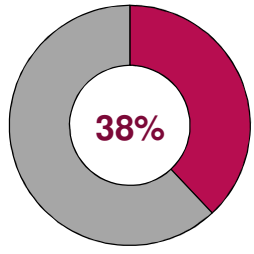

... of VCSE organisations felt local authorities are a positive influence on their success, almost identical to the proportion in 2012/13; but more respondents (51 per cent) also again felt their most frequent other public sector contact was a positive influence on their success

\section{Q9. How well does the VCSE sector work with private businesses?}

\section{$62 \%$ had some dealings with local private businesses}

... 31 per cent of respondents felt that the private business community in Greater Manchester was a positive influence on their organisation's success - this is an increase of 12 percentage points since $2012 / 13$

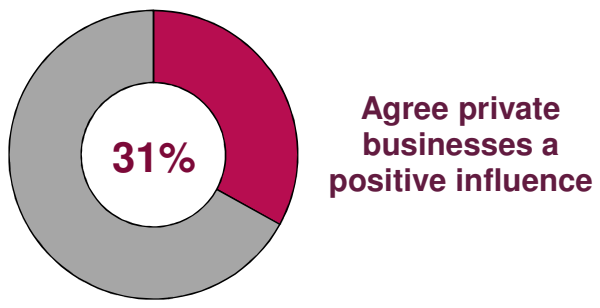

\section{$2 \%$ are members of a private sector-led consortium}

\section{Q10. How well does the VCSE sector work together?}

$67 \%$ had a 'great' or 'fair amount' of contact with other vCSE organisations in their local area (37 per cent with VCSE organisations in other parts of Greater Manchester)
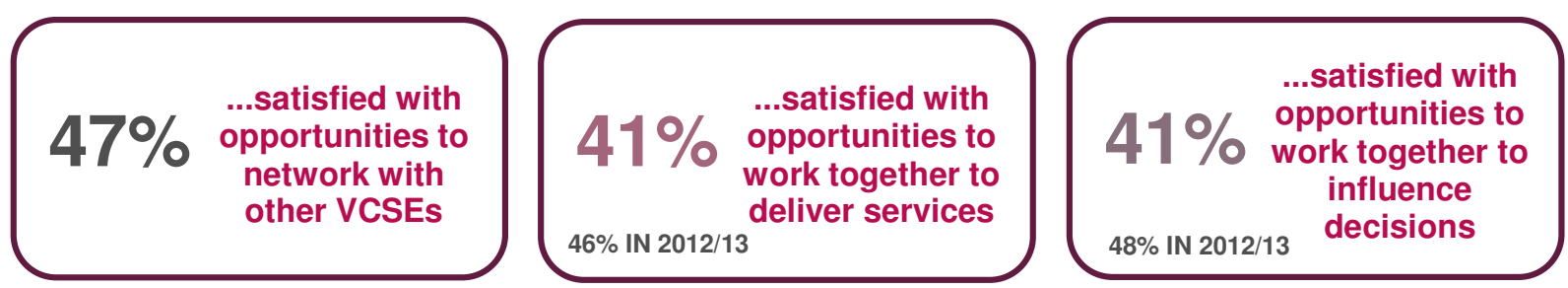

$22 \%$ are members of a formal VCSE consortium 


\section{Q11. What are the key issues facing the VCSE sector in the future?}

Respondents were asked about the strategies they are actively pursuing or planning to pursue. 56 per cent of organisations or more were already doing or planning to do the following:

\section{$64 \%$ work more closely with another voluntary/not-for-profit organisation $60 \%$ increase earned income $56 \%$ change the way they run services or activities}

Respondents were also asked to consider the factors they anticipated assisting or constraining their organisation over the next 12 months:

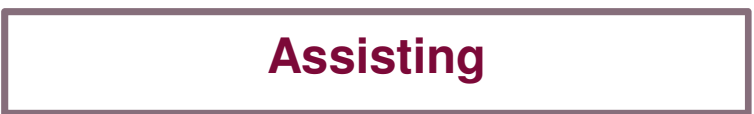

TOP FACTORS:

Engagement with other VCSE organisations (50 per cent assisting or greatly assisting)

Engagement with public sector bodies (48 per cent)

Ability to secure other sources of income (47 per cent)

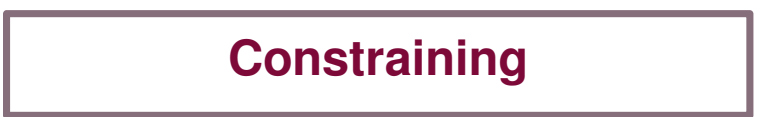

\section{TOP FACTORS:}

The local economy (38 per cent constraining or seriously constraining)

Ability to recruit volunteers with sufficient skills ( 31 per cent) 


\section{Introduction}

This report provides the main findings of research aimed at improving the understanding of the social and economic impact of the voluntary, community and social enterprise (VCSE) sector in Greater Manchester. The research was commissioned by GMCVO, 10GM ${ }^{5}$ and Stockport Council and undertaken by the Centre for Regional Economic and Social Research (CRESR) at Sheffield Hallam University.

The key objective of the research was to provide a comprehensive overview of the sector in Greater Manchester at the start of 2017 and to show some of the ways it has changed since the previous survey of 2013.

The research involved a web-based survey of organisations supporting the people in Greater Manchester and focus groups with VCSE organisations in five Greater Manchester districts. The research took place between September 2016 and January 2017.

Appendix 1 provides further detail on the research methodology.

\section{Key findings from the research}

\section{The VCSE sector in Greater Manchester continues to occupy an important strategic position within the city region.}

There are an estimated 15,890 organisations working in the VCSE sector in Greater Manchester which are involved in many areas of activity.

As in the 2013 study, the local geographic area is a main focus for the majority of organisations; 34 per cent identified particular neighbourhoods or communities in Greater Manchester as their highest main geographic focus and a further 27 per cent identified one particular local authority area as their highest main geographic focus.

The thematic areas with the greatest proportion of organisations working in them are: health and wellbeing; community development; education, training and research; and sport and leisure; the same four areas were selected most frequently in 2012/13.

\footnotetext{
${ }^{5} 10 \mathrm{GM}$ is a joint venture by the Greater Manchester Voluntary Sector Infrastructure Organisations including Salford CVS (lead partner on this research), Action Together in Oldham and Tameside, Bolton CVS, CVS Rochdale, Macc and Wigan and Leigh CVS.
} 
The VCSE sector plays a key role in fostering strong and cohesive communities within Greater Manchester and is an essential part of the social and economic fabric of the city region. Over two-thirds (68 per cent) of organisations feel they are improving people's mental wellbeing, while over three-fifths (63 per cent) stated they are addressing the needs of disadvantaged members of the community and 60 per cent see themselves as increasing people's skills.

2. The sector in Greater Manchester remains an important economic player, contributing significantly to $G V A^{6}$, but patterns in income, expenditure and the level of reserves suggest that, as in 2013, the sustainability of many organisations may be under threat.

Total income of the VCSE sector in $2014 / 15$ is estimated to be $£ 1.321 \mathrm{bn}$. This represents an increase of three per cent compared to 2013/14 when the total income of the VCSE sector was an estimated $£ 1.281$ million. The vast majority of organisations are micro or small although the majority of income is concentrated in large and medium-sized organisations.

The picture is more positive overall than in the previous 2013 study which identified year-on-year reductions in income. However, analysis of income data by organisation size revealed micro and small organisations experienced year on year reductions in total income between 2012/13 and 2014/15. By contrast medium and large organisations saw a reduction in total income between 2012/13 and 2013/14 but then an increase between 2013/14 and 2014/15.

Half the respondents reported increasing their expenditure but only 40 per cent had experienced an increase in income and only 26 per cent report an increase in reserves. In addition, 28 per cent of respondents reported a decrease in income but only 16 per cent reduced their expenditure.

23 per cent of respondents provided an expenditure figure for 2014/15 that was greater than their income. These results indicate a sizeable number of organisations spent more money than they received in the last 12 months and that a considerable number of organisations are using their reserves to supplement their income, potentially leaving them in a fragile financial position.

\section{The VCSE sector in Greater Manchester continues to provide significant social value.}

It is estimated that the VCSE sector in Greater Manchester made 21.9 million interventions with clients, users or beneficiaries in the previous year.

VCSE organisations work with a range of different people, especially children and young people, older people, but also people experiencing disadvantage (for example those with health problems and unemployed people).

\section{The VCSE sector continues to be a significant employer.}

In 2016/17 there were an estimated 28,600 FTE paid staff. In addition the sector was supported by 355,800 volunteers and 105,900 committee/board members who combined donated 1.1 million hours per week.

\footnotetext{
${ }^{6}$ Gross Value Added (GVA), the value of goods and services produced, is a key measure of the economic contribution of organisations or sectors.
} 
Valuing the contribution of paid employees to Greater Manchester organisations by the expected value of the output that they produced gives an estimated annual contribution of $£ 905.3$ million.

\section{Volunteering is an essential and highly valuable feature of the 'identity' of the VCSE sector, however there are challenges associated with volunteering across Greater Manchester.}

Focus group participants highlighted volunteering as an essential and highly valuable feature of the 'identity' of the sector and its work supporting people and communities. A range of benefits were consistently identified with volunteering: innovation and skills; service delivery; reach; governance and accountability; active citizenship; and a pathway to employment.

Valuing the contribution of volunteers and committee/board members to Greater Manchester organisations by the expected value of the output that they produced gives an estimated annual contribution of $£ 973.0$ million.

Over two-fifths (42 per cent) of organisations responding to the survey reported increased numbers of volunteers compared to the previous year while 13 per cent of organisations reported a decrease in volunteer numbers.

The focus groups provided a mixed picture of whether or not levels of volunteering had changed in recent years. In general larger VCSE organisations were more likely to have reported an increase in volunteers, whilst smaller organisations were more likely to be struggling to retain and recruit volunteers.

Focus group participants also identified a number of challenges associated with volunteering. Foremost amongst these was the need for 'quality volunteers'.

\section{There is a mixed picture in Greater Manchester regarding relationships between the VCSE sector and public sector bodies.}

Overall, 84 per cent of respondents in Greater Manchester had some dealings with at least one local authority (an increase from 72 per cent in 2012/13): 16 per cent had a great amount of dealings with at least one local authority and 36 per cent had a fair amount of dealings.

Less than one third (30 per cent) of respondents were satisfied with their ability to influence local authorities' decisions of relevance to their organisation while 38 per cent said local authorities had a positive influence on their organisation's success, similar to the proportions reported in 2013.

VCSE organisations who participated in focus groups were critical of local public sector bodies and the way they work with VCSE organisations, highlighting a growing sense that VCSEs and the state are becoming increasingly 'disconnected'. Participants also expressed uncertainty about the implications of devolution for their work and many were sceptical and cautious that it would be a positive development.

Participants were however keen to highlight pockets of good practice within the public sector in its dealings with VCSEs and emphasised that relationships and practices tended to vary over time.

7. Engagement with private businesses has increased and perceptions of the private business sector appear to have improved, however engagement is still relatively low. 
62 per cent of organisations had some direct dealings with private businesses, with 21 per cent having a 'great' or 'fair' amount of contact. This is an increase since 2012/13 when 55 per cent reported some direct dealings, including 16 per cent having a 'great' or 'fair' amount of contact.

Almost one third (31 per cent) felt that the private business community in Greater Manchester was a positive influence on their organisation's success. This is an increase since 2012/13 when just 19 per cent agreed private businesses were a positive influence.

Overall, focus group participants viewed working with private businesses positively, particularly when it meant forging links with local business with strong roots in their area.

However, participants also identified barriers to partnership working with the private sector. These included a 'cultural divide' between the two sectors, and the perception that they have fundamentally different value bases.

\section{The VCSE sector in Greater Manchester continues to be well connected internally although most contact appears to be informal.}

As in the 2013 study, the vast majority of organisations had some direct dealings with other VCSE sector organisations in their local area, including 67 per cent who had a 'great' or 'fair amount' of contact.

22 per cent of respondents said their organisation is a member of a formal VCSE sector consortium.

Focus group participants were overwhelmingly positive about working in partnership with other VCSE organisations and its importance moving forward. Strong personal relationships with key staff in other VCSE organisations, embodied by high levels of trust, were consistently identified as a key factor in enabling effective partnership working.

Where partnership working had been less effective this was often due to 'mismatches' between organisations.

\section{The sector still faces an uncertain future.}

With austerity measures set to continue for the foreseeable future and public sector funding for the sector continuing to be squeezed, there are still reasons for caution within the sector.

Respondents appear to recognise this uncertainty and are pursuing a range of strategies to ensure their sustainability, in particular: generating earned income from other sources, partnership working and organisational change. 


\section{Context for the Research}

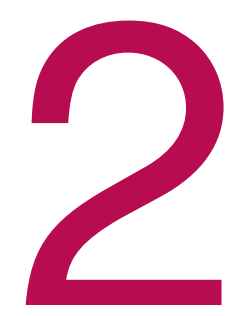

This research comes during both a period of slow economic recovery following the long-term economic downturn and a rapidly changing political backdrop as devolution in Greater Manchester gains pace.

With the election of the Conservative Government in May 2015, austerity measures are set to continue for the foreseeable future and VCSE organisations are likely to feel the impact of these measures. This includes the Government's commitment to a continuing programme of welfare reform which is likely to result in increasing demand for some services as benefits are restricted or withdrawn. The total anticipated reduction by $2020 / 21$, from both pre and post-2015 welfare reforms in Greater Manchester, is predicted to be $£ 540 \mathrm{~m}$ per year or equivalent to $£ 680$ per working age adult per year. ${ }^{78}$ These reforms are likely to continue to put pressure on VCSE organisations both in terms of their financial health and the need to meet greater levels of need from existing and new beneficiaries.

Encouragingly people still appear able and willing to engage in voluntary action. There are increasing numbers of charitable interest companies and social and community enterprises and entrepreneurs in Greater Manchester. Less positively, the number of food banks has also continued to rise across the region.

Greater Manchester local authorities have experienced, and are continuing to experience, a decline in Government funding. Because public spending cuts are correlated with deprivation, Greater Manchester local government has been particularly badly affected. Bury, Trafford and Stockport are especially affected, since they did not benefit in previous years from additional targeted funding from central government.

However, the harshest impact of cuts may be partially offset by increasing levels of devolution, enabling greater local control of decisions and increased collaboration across Greater Manchester agencies. In this context there is increasing interest amongst public and private organisations in collaboration with the VCSE sector, and in its current and potential role within a devolved and devolving city region.

Against this background this research provides in depth data about the 'state of the VCSE sector' in Greater Manchester at the start of 2017. The research provides a comprehensive overview of the sector in Greater Manchester for partners to draw upon and help harness the potential of the sector.

\footnotetext{
${ }^{7}$ Beatty and Fothergill (2016) The Uneven Impact of Welfare Reform: The financial losses to places and people.

${ }^{8}$ Note: These figures are based on HMRC Budgets and Autumn Statements from 2010 to 2015. In the 2016 Autumn statement the Pay-to-stay measure was scrapped and so this has been taken account of in the figures. The estimate of cuts due to the LHA Cap in social housing was increased by a further $£ 160 \mathrm{~m}$ p.a. which is not taken account of in the figures. The Universal Credit Taper was also increased by $2 p$ in the pound, an increase in funding of $£ 570 \mathrm{~m}$ p.a., which is not included in the figures presented here.
} 


\section{What the Voluntary Community and Social Enterprise Sector in Greater Manchester does}

This chapter develops a picture of the core features of the voluntary, community and social enterprise (VCSE) sector in Greater Manchester. It focuses on a series of general questions in which respondents were asked about their group or organisation: what it is and what it does.

\subsection{How many VCSE organisations are there in Greater Manchester?}

Estimating the number of organisations represents a major challenge. This is because a large proportion of organisations are small, local and not formally constituted as charities, limited companies or other recognised forms which require registration (e.g. industrial and provident societies). As a result they do not appear on formal central records such as those held by the Charity Commission or Companies House so are considered 'below the radar' (BTR). Any estimate of the total number of organisations in an area therefore requires information on the numbers of registered and unregistered (i.e. BTR) organisations.

In estimating the total number of organisations in Greater Manchester we drew on information from the following sources:

- The Register of Charities in England and Wales, which indicated 4,279 registered charities with postcodes in Greater Manchester.

- The ratio of charities to non-charities provided in the 'National Survey of Charities and Social Enterprises' (NSCSE), undertaken by Ipsos MORI for the Cabinet Office in 2010. This was used to gross the estimate upwards to a total of 5,802 registered organisations, to take account of non-charitable social enterprises.

- Research by NCVO and the University of Southampton ${ }^{9}$ which found that on average there are $3.66 \mathrm{BTR}$ organisations per 1,000 population. If this figure is applied to Greater Manchester ${ }^{10}$, it can be estimated that there are 10,088 BTR organisations in the city region. ${ }^{11}$

\footnotetext{
${ }^{9}$ Mohan, J et al. (2010). Beyond 'flat-earth' maps of the third sector: enhancing our understanding of the contribution of 'below-the-radar' organisations. Northern Rock Foundation Briefing Paper

${ }_{11}^{10}$ Based on Office for National Statistics 2015 population estimates

${ }^{11}$ It is important to note that the BTR figure is an estimate based on an average across 46 local authorities. The BTR research found significant variability, with some local authorities reaching over seven BTR organisations per 1,000 population, and in one case exceeding 10.
} 
Summing the estimated numbers for both registered and BTR organisations produces an estimated figure of:

\section{5,890 organisations in total operating in the VCSE sector in Greater Manchester}

A breakdown by area is provided in table 3.1.

Table 3.1: Estimated number of organisation in the sector in Greater Manchester

\begin{tabular}{lrrr}
\hline & $\begin{array}{c}\text { No of } \\
\text { organisations }\end{array}$ & Registered & \multicolumn{1}{c}{ BTR } \\
\hline Bolton & 1,561 & 530 & 1,031 \\
Bury & 1,135 & 447 & 688 \\
Manchester & 3,394 & 1,453 & 1,941 \\
Oldham & 1,231 & 386 & 845 \\
Rochdale & 1,180 & 396 & 784 \\
Salford & 1,513 & 614 & 899 \\
Stockport & 1,689 & 632 & 1,057 \\
Tameside & 1,167 & 355 & 811 \\
Trafford & 1,455 & 601 & 854 \\
Wigan & 1,566 & 387 & 1,179 \\
\hline Total & $\mathbf{1 5 , 8 9 0}$ & $\mathbf{5 , 8 0 2}$ & $\mathbf{1 0 , 0 8 8}$ \\
\hline
\end{tabular}

This figure represents an increase of nine per cent since the estimate produced for the 2013 report $(14,592)$. This may reflect a genuine increase in the number of voluntary organisations between the two surveys, but could also be due to unavoidable differences in the estimation methodology.

For the 2013 report, the sampling frame for the NSCSE was used to provide the estimates for the number of formally registered organisations. Unfortunately this survey was subsequently cancelled and has not been repeated. As such, only the ratio of charities to non-charities was taken from this data source and combined with the number of charities from the charity register.

\subsection{What size are organisations in Greater Manchester?}

The size of organisations is traditionally measured using their annual income ${ }^{12}$. When the distribution of organisations across Greater Manchester was explored by size category based on income for 2014/15, it showed that the majority of organisations were either micro or small. But the survey was underrepresentative of BTR organisations (only 33 per cent of survey respondents were identified as BTR), so this did not present an accurate picture of the actual distribution. The figures were therefore adjusted based on the assumption that the estimated 8,006 organisations not included in the survey sample were BTR and micro in size ${ }^{13}$.

Exploring the distribution by size category based on income for $2014 / 15$ also showed some inconsistency with the distribution found in 2013. Therefore, in order to provide

\footnotetext{
${ }^{12}$ In exploring organisation size we used the categories developed by NCVO for use in their Almanac series (see e.g. Clark, J et al., 2010)

${ }_{13}$ The basis for these assumptions is discussed in more detail in the methodological annex
} 
the most robust estimate of the distribution of organisations in the VCSE sector by size, data from both waves of the survey have been used to calculate the proportion of organisations estimated to be in each size category.

The outcome of this process is shown in figure 3.1 , which demonstrates that an estimated 77 per cent of the VCSE sector (12,312 organisations) are micro in size, 12 per cent are small (1,983 organisations), eight per cent are medium $(1,221$ organisations), and two per cent are large (374 organisations).

Introducing the BTR figure produces a much higher estimate for the number and proportion of micro organisations and emphasises the finding that a large proportion of organisations in the VCSE sector in Greater Manchester are very small (90 per cent micro or small). This is similar to the national picture: $\mathrm{NCVO}^{14}$ estimate that 83 per cent of the VCSE sector is made up of micro or small organisations, 14 per cent are medium, and three per cent are large.

Figure 3.1: Proportion of Greater Manchester VCSE organisations by size (estimated)

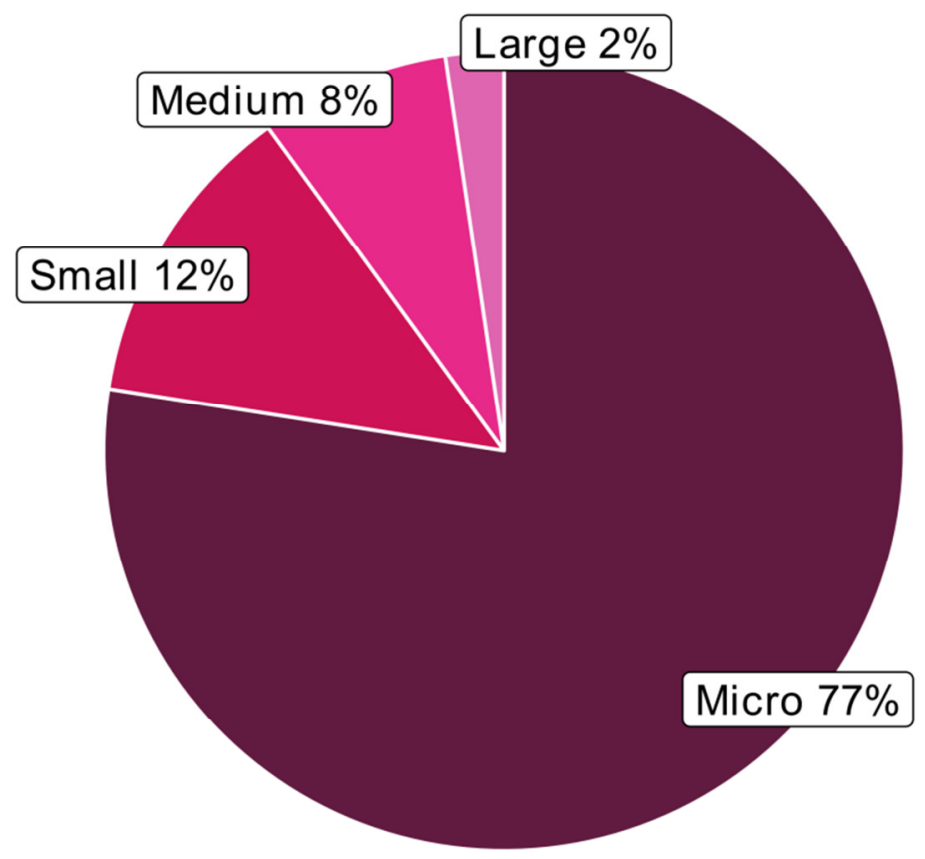

Source: Greater Manchester State of the VCSE sector survey 2016/17 Base: 720

\subsection{What types of organisations operate in the VCSE sector in Greater Manchester?}

Respondents to the questionnaire were asked to identify which category from a list of 'organisation types' best described their organisation. The results indicate that many organisations in the VCSE sector are likely to have a local focus. Figure 3.2 shows that the largest proportion, 22 per cent, identified their organisation as being a local voluntary organisation. This proportion is some seven percentage points higher than for the next most common type: community or neighbourhood group (15 per cent). National organisations were less common: just over one in ten organisations stated they were either a national voluntary organisation (two per cent),

\footnotetext{
${ }^{14}$ UK Civil Society Almanac (2016) NCVO.
} 
a branch of a national voluntary organisation (six per cent) or an affiliated member of a national voluntary organisation (four per cent).

This breakdown of organisations by type followed a similar pattern to that in the $2012 / 13$ survey, where the two largest categories were local voluntary organisation (26 per cent) and community or neighbourhood group (18 per cent). National organisations were also less common in 2012/13.

Figure 3.2: Type of organisations

Source: Greater Manchester State of the VCSE sector survey 2016/17

Base: 1,156

\subsection{How long have organisations in the VCSE sector been operating?}

The questionnaire asked respondents to indicate when their organisation was formed. Assessment of organisations by the year in which they were formed provides an indication of how established the VCSE sector is in Greater Manchester. Figure 3.3 details the responses received to this question. It is important to highlight that the figures displayed only provide information on surviving organisations. Therefore although the results suggest that it is likely that the VCSE sector in Greater Manchester has experienced growth in the number of organisations established in the last 20 years or so, it may not be as dramatic as the figures suggest.

The responses received build a picture of a VCSE sector that has a fairly well established core. However, the VCSE sector in Greater Manchester has also seen the formation of many new organisations since 2001. Figure 3.3 shows that 54 per 
cent of organisations responding to the survey had been formed since 2001, including 43 per cent in the past 10 years (i.e. since 2006). Almost one third (29 per cent) of organisations had been formed since 2011. Furthermore, an additional 13 per cent were formed between 1991 and 2000; this means two-thirds (67 per cent) of organisations were formed in the last 25 years. At the other end of the spectrum 16 per cent of organisations had been formed before 1971, including six per cent formed in 1910 or before.

In the 2012/13 survey, 62 per cent of organisations were formed since 1991, including 37 per cent, which had been formed in the past 10 years. Seven per cent of organisations in 2012/13 had been formed in 1910 or before.

This pattern was broadly similar across the seven Greater Manchester areas.

Figure 3.3: Year in which organisations were formed

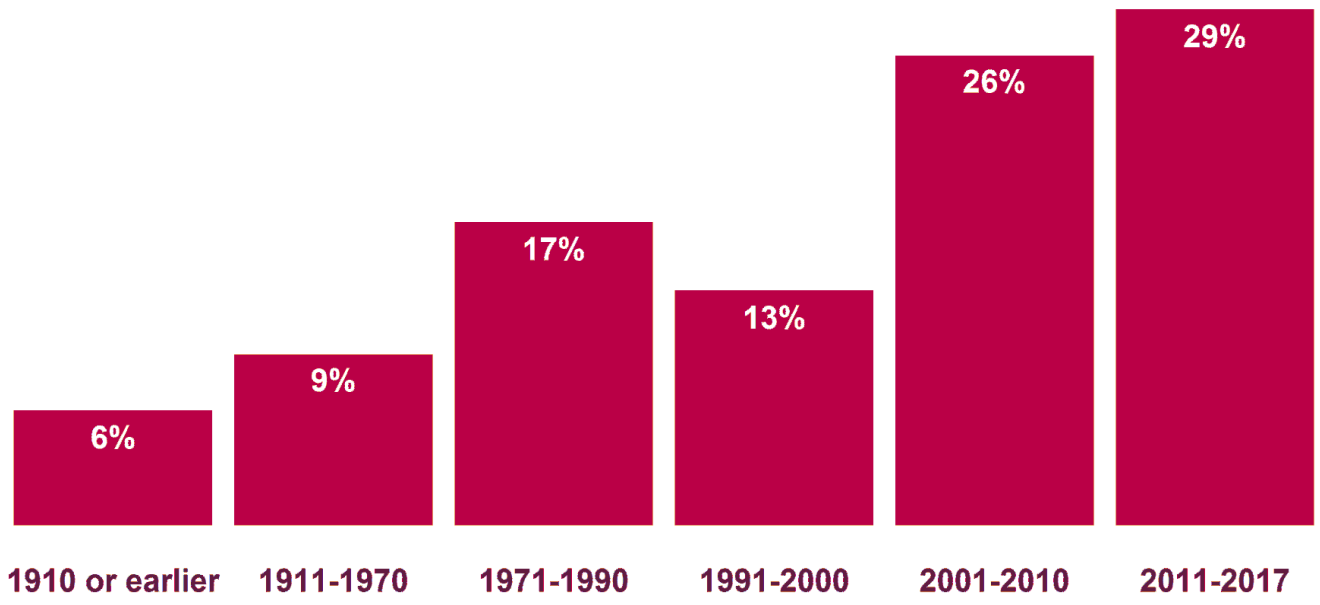

Source: Greater Manchester State of the VCSE sector survey 2016/17 Base: 1,056

\subsection{What does the VCSE sector in Greater Manchester do?}

To elicit a picture of what the VCSE sector in Greater Manchester does, the survey asked respondents to identify up to three main thematic areas in which their organisation operates. Figure 3.4 presents the top ten main areas selected and confirms the message that the VCSE sector in Greater Manchester works in a diverse range of thematic areas. However, the proportion of responding organisations working in each area varies. This is most likely dependent on demand and funding opportunities.

Figure 3.4 shows:

- $\quad 46$ per cent of organisations worked in the area of health and well-being; in $2012 / 13$ this was also the most common main area (37 per cent of organisations)

- $\quad 39$ per cent worked in community development, similar to the $2012 / 13$ survey (37 per cent)

- $\quad$ over one quarter of organisations worked in each of the following themes:

- $\quad$ education, training and research (26 per cent compared to 28 per cent in 2012/13)

- $\quad$ sport and leisure (25 per cent compared to 27 per cent in 2012/13). 
Analysis across the seven study areas finds a similar pattern within each local authority area with the same four areas of work featuring as the most common except for in the City of Manchester, where economic well-being was the fourth most common area of work and sport and leisure was the fifth.

Figure 3.4: Top 10 main areas in which organisations work

Health and well-being

Community development

Education, training and research (including information, advice and guidance)

Sport and leisure

Economic well-being

Arts, heritage and culture

Social care

Capacity building and other support for voluntary or community organisations

Environment and sustainability

Other charitable, social or community purpose

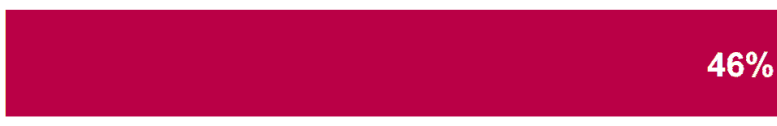

$39 \%$
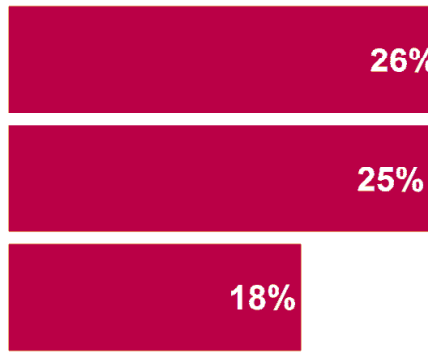

$13 \%$

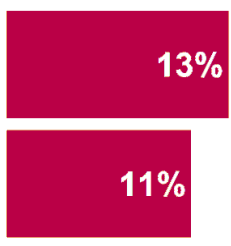

$10 \%$

$10 \%$

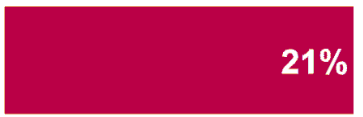

Source: Greater Manchester State of the VCSE sector survey 2016/17

Base: 1,155

In the latest survey respondents who indicated they worked in the area of health and well-being were asked to specify the specific areas in which they operate. The majority (80 per cent) stated they worked in health and well-being in general. Over half (55 per cent) indicated they worked in mental health. In addition over one third said they worked in healthy living (food \& lifestyle, sexual health) (37 per cent).

In a similar vein, respondents who identified education, training and research as a main area of work were asked to specify the areas they worked in within this theme. Of the respondents who answered this question, 66 per cent stated they worked in education, while 64 per cent said they worked in information, advice and guidance. Three-fifths (60 per cent) indicated they worked in the area of employability skills.

The five per cent of organisations responding to the survey who indicated that equalities and civil rights was a main theme of their work were also asked to identify the specific areas within this category in which they operate. Two-thirds (66 per cent) stated they worked in the area of disability, while over half of respondents gave age or gender as a response (57 per cent and 55 per cent respectively). 


\section{Who the Voluntary}

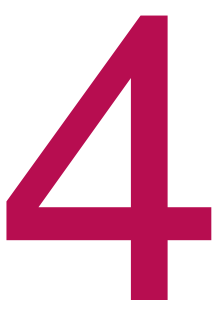

Community and Social Enterprise Sector in Greater Manchester works with

This chapter focuses on who the VCSE sector in Greater Manchester works with and where.

4.1. Who are the clients, users or beneficiaries of the VCSE sector in Greater Manchester?

The questionnaire asked respondents to provide the total number of individual clients, users or beneficiaries that their organisation had supported in the last year. Analysis of responses to this question by size and type of organisation revealed that in many cases organisations had provided the number of 'interventions' or 'contacts' that they had had with clients, users or beneficiaries. So for example an individual who visited a community centre once a week would have been counted 52 times within the year. Whilst some organisations will have provided the number of unique clients, users or beneficiaries, so as not to overestimate, in our analysis we have assumed the number provided represents the total number of interventions.

Summing across the 992 organisations that responded gives a total of 4.5 million interventions overall. The responses received can be extrapolated for the estimated 15,890 organisations thought to be operating in the VCSE sector in Greater Manchester to provide an estimate of the total number of interventions by Greater Manchester organisations. Working through the calculation it is estimated that Greater Manchester organisations had made:

\section{9 million interventions with clients, users or beneficiaries in the past year overall}

The 2012/13 study estimated that Greater Manchester organisations made 21.2 million interventions with clients, users or beneficiaries overall.

The questionnaire also asked respondents to identify up to three groups that make up the main clients, users or beneficiaries of their organisation. Figure 4.1 shows that, as might be expected, the VCSE sector in Greater Manchester serves a diverse and wide ranging population. In many cases, client groups are served by relatively small numbers of organisations: 10 per cent of organisations or fewer serve 17 of the client groups listed. 
Figure 4.1 shows that the client groups served by the largest proportions of organisations can be broadly characterised as being demographic: gender - women (15 per cent) and men (12 per cent) - and age - children and young adults (23 per cent) and older people (17 per cent).

General and demographic client groups were also the most common groups identified in the 2012/13 survey, although the ordering was different. In 2012/13 the most common client groups were: women (26 per cent), young people (24 per cent), children (24 per cent), men (23 per cent) and older people (23 per cent).

One third (33 per cent) of organisations identify 'everyone' as their main clients, users or beneficiaries. Individuals with health issues are also served by relatively high proportions of organisations. Disabled people are a main client, user or beneficiary group for 10 per cent of organisations and people with mental health problems also make up a main client, user or beneficiary group for 10 per cent of organisations. In the 2012/13 survey these proportions were 14 per cent and 11 per cent respectively.

Analysis of responses from each of the Greater Manchester areas found a similar pattern. General and demographic client groups and individuals with health issues were the most common beneficiary groups identified. The picture was slightly different in the City of Manchester where Black and Minority Ethnic communities and unemployed people were among the most common beneficiary groups identified. 
Figure 4.1: Top 10 main client groups of Greater Manchester organisations

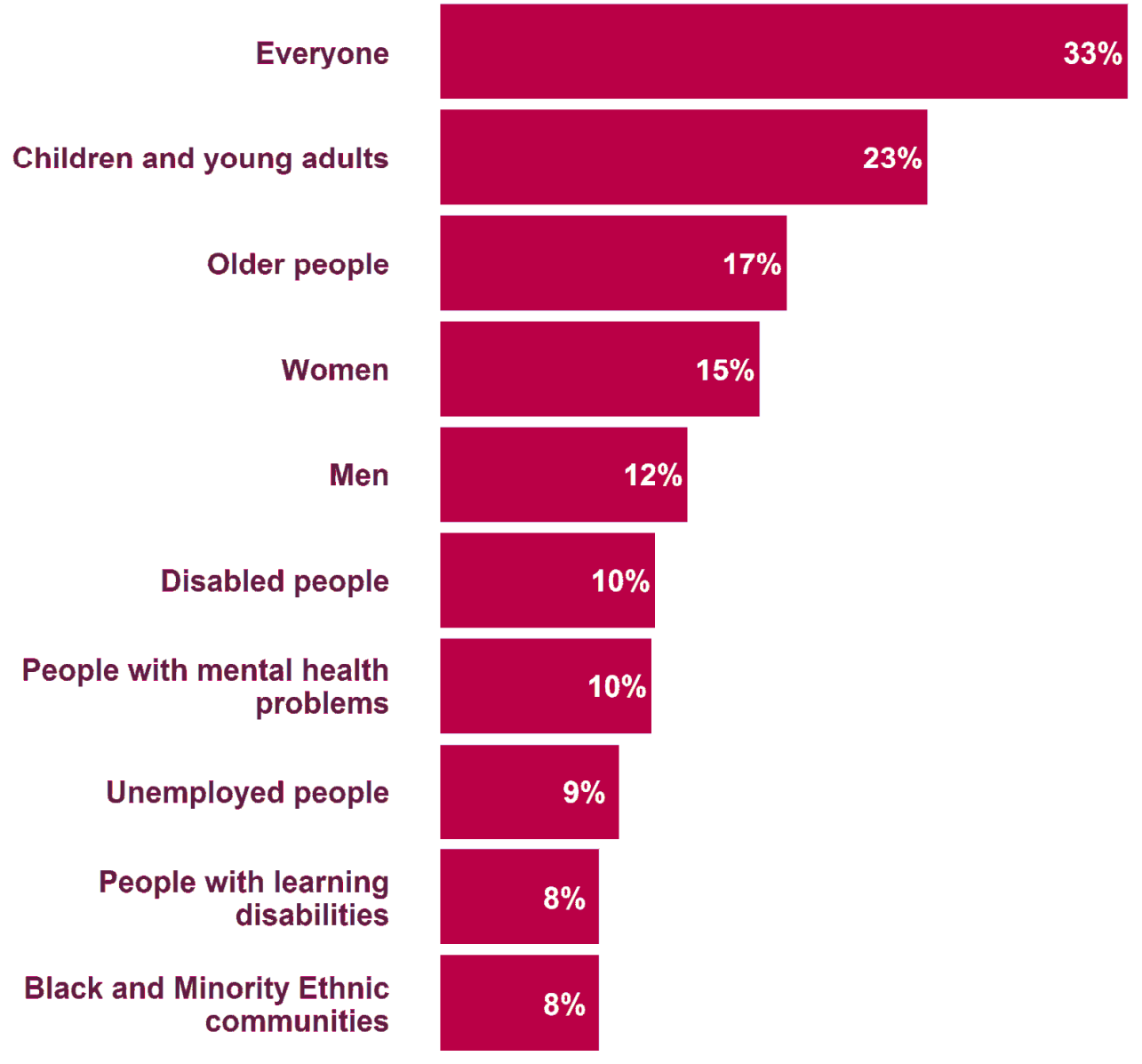

Source: Greater Manchester State of the VCSE sector survey 2016/17 Base: 1,159

Respondents to the 2016/17 survey were asked to identify the ways in which their organisation makes a difference for its service users/client group(s). This question demonstrates the key role that the VCSE sector has in fostering strong and cohesive communities within Greater Manchester and highlights the importance of the VCSE sector as an essential part of the social fabric of the city region. As figure 4.2 shows, over two-thirds (68 per cent) of organisations felt they are improving people's mental wellbeing, while over three-fifths (63 per cent) stated they are addressing the needs of disadvantaged members of the community and 60 per cent saw themselves as increasing people's skills. 
Figure 4.2: Top 10 ways in which organisations make a difference

Improving people's mental wellbeing

Addressing the needs of disadvantaged members of the community

Increasing people's skills

Encouraging people to be involved in regular volunteering

Improving people's physical wellbeing

Helping people to feel that they belong to their neighbourhood

Encouraging people to get involved in activities and events that are happening

Helping people from different backgrounds to get on well together

Helping people to feel safe

Helping people feel that Greater Manchester is a good place to live

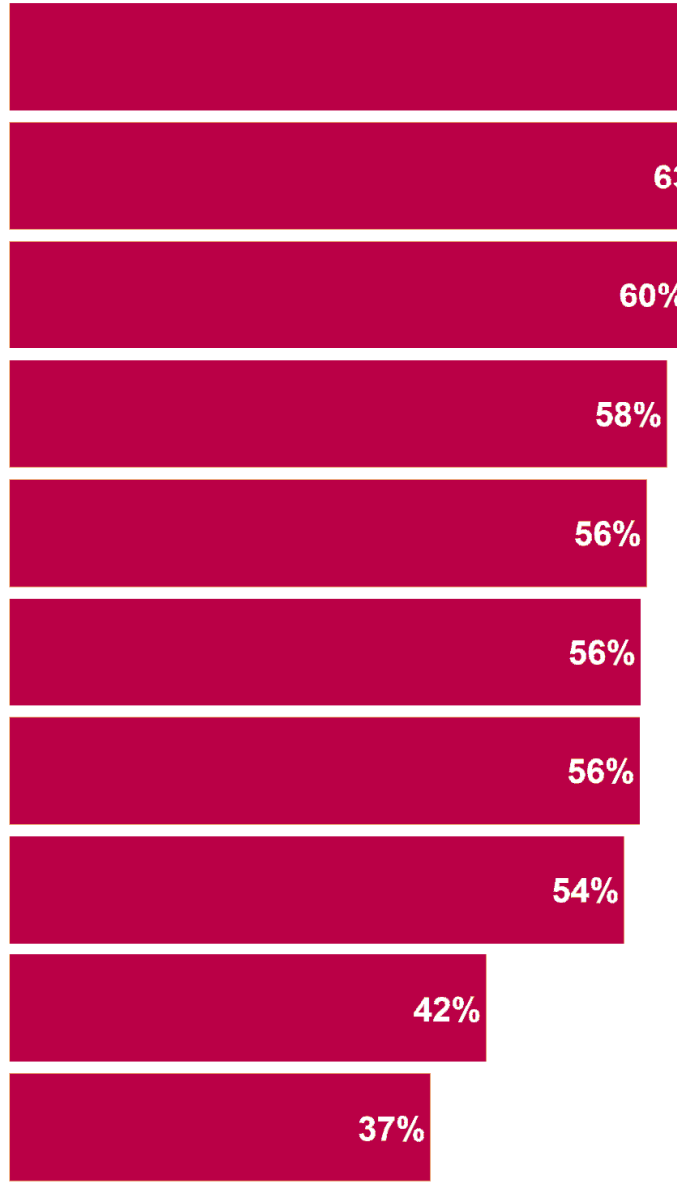

Source: Greater Manchester State of the VCSE sector survey 2016/17

Base: 1,158

\subsection{What geographical levels does the VCSE sector operate at?}

The survey asked respondents to identify the main geographical levels at which they operate - this ranged from the neighbourhood level, to those operating across England, the UK or overseas ${ }^{15}$. In this question respondents were asked to pick out up to three main geographic levels, the results of which are presented in figure 4.3. This shows that the local area is a main focus for a majority of organisations:

- over two-fifths (44 per cent) identified particular Greater Manchester neighbourhoods or communities as a main focus; a lower proportion than in 2012/13 (57 per cent)

- a further 40 per cent identified one particular local authority area as a main focus; in the 2012/13 survey 42 per cent identified particular local areas in Greater Manchester as a main focus (respondents in 2012/13 weren't asked to identify if they worked in one or more than one local authority area).

\footnotetext{
${ }^{15}$ This question was asked slightly differently in the latest survey compared to 2012/13. Two additional options ('Across more than one Greater Manchester Local Authority area' and 'Across the whole of Greater Manchester') were included.
} 
A modest proportion of organisations cited that a main geographic area at which they work was either national (11 per cent) or international (three per cent). In many cases those organisations that work internationally will reflect their main clients, users and beneficiaries.

Figure 4.3: Main geographic focus

Particular

Greater Manchester neighbourhoods and communities

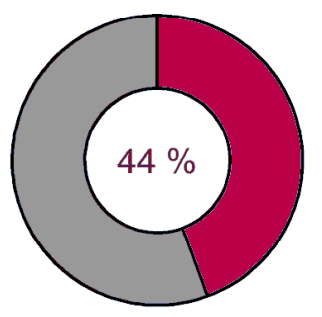

Regionally across the North West

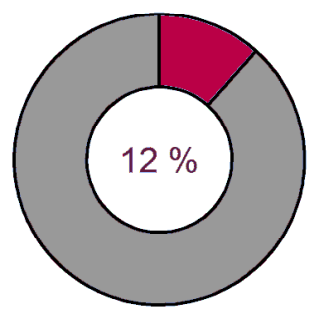

\section{Across one Greater Manchester Local Authority area}

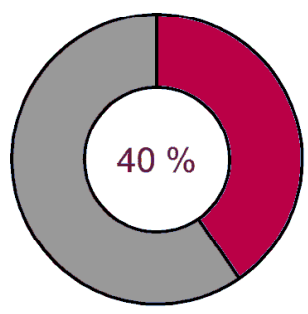

Nationally

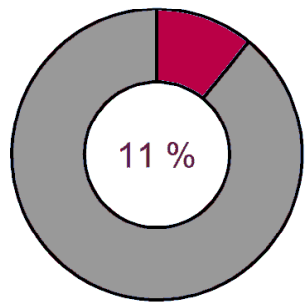

Across more than one Greater Manchester Local Authority area

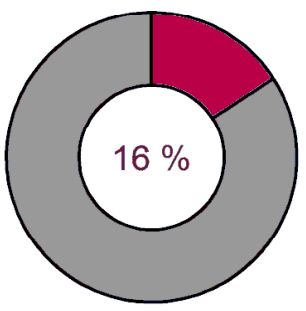

Internationally

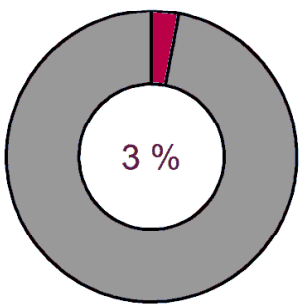

Across the whole of Greater Manchester

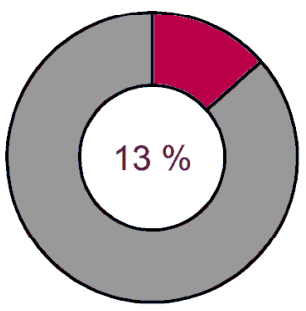

Source: Greater Manchester State of the VCSE sector survey 2016/17

Base: 1,156

Using the responses to this question it is also possible to identify the highest main geographic area that an organisation carries out its activities (see figure 4.4 below). The highest geographic area that could be identified was internationally.

This analysis finds that for over one third (34 per cent) their highest main geographic focus was particular Greater Manchester neighbourhoods or communities; a smaller proportion than in 2012/13 (41 per cent). 
Figure 4.4: Highest geographic focus

Particular

Greater Manchester neighbourhoods and communities

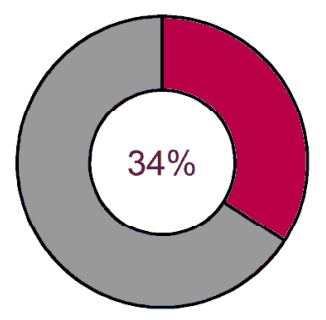

Regionally across the North West

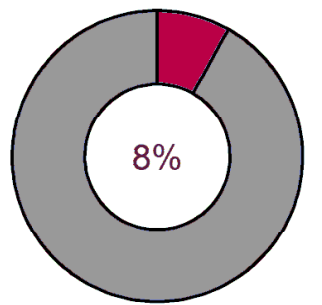

Across one Greater Manchester Local

Authority area

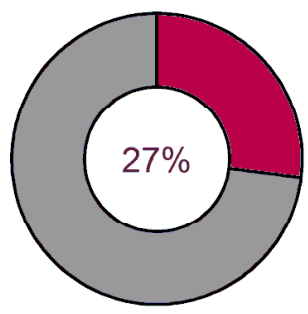

Nationally

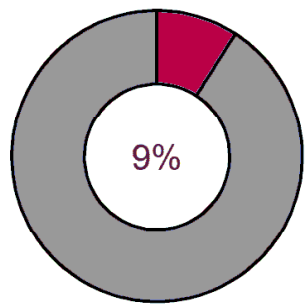

Across more than one Greater Manchester Local Authority area

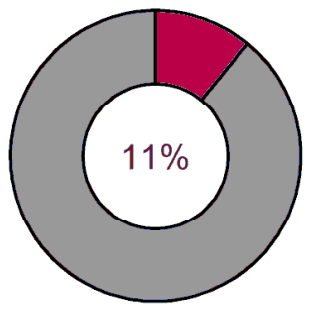

Internationally

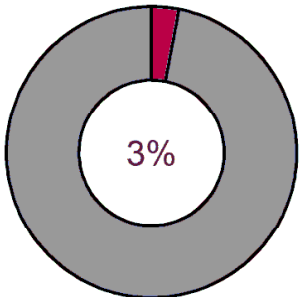

Across the whole of Greater Manchester

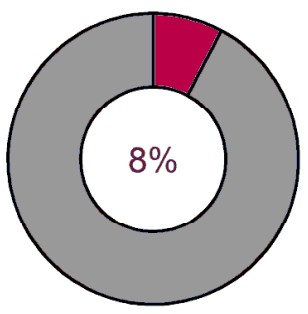

Source: Greater Manchester State of the VCSE sector survey 2016/17

Base: 1,156 


\section{Finances and Income}

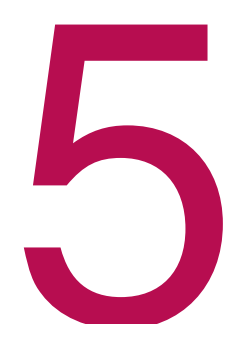

This chapter provides an overview of the finances and income of the VCSE sector in Greater Manchester. It includes estimates of the overall income received by the sector between 2012/13 and 2014/15, analysis of the different sources of income received (public sector and non-public sector) and their relative contribution, and an assessment of the financial sustainability of the VCSE sector.

Where possible this chapter compares results from the latest survey and the 2012/13 study. Revisions to the questionnaire and methodology between these studies, however, mean that comparisons are not always possible or appropriate and that caution should be applied when comparing across the two waves (see Appendix 1 for more detail).

\subsection{Income}

Based on the average (mean) income of respondents to the survey across Greater Manchester, and drawing on the assumptions used to estimate the total number of organisations in Greater Manchester, the following is estimated ${ }^{16}$ -

\section{$£ 1.321$ billion the total income of the VCSE sector in Greater Manchester in 2014/15}

This total income estimate is higher than the figure of $£ 1$ billion estimated as being the total income for the sector in 2011/12 from the 2012/13 survey. It also represents an increase of three per cent compared to 2013/14 when the total income of the VCSE sector was an estimated $£ 1.281$ million. This follows a reduction between $2012 / 13$ and $2013 / 14$ of an estimated six per cent in the total income of the sector. This data is outlined in more detail in figure 5.1.

\footnotetext{
${ }^{16}$ This figure is based on a weighted average (mean) for each size category for respondents from across Greater Manchester. The methodology is explained in more detail in the methodological appendix.
} 
Figure 5.1: Estimated annual income of the VCSE sector in Greater Manchester (2012/13-2014/15)

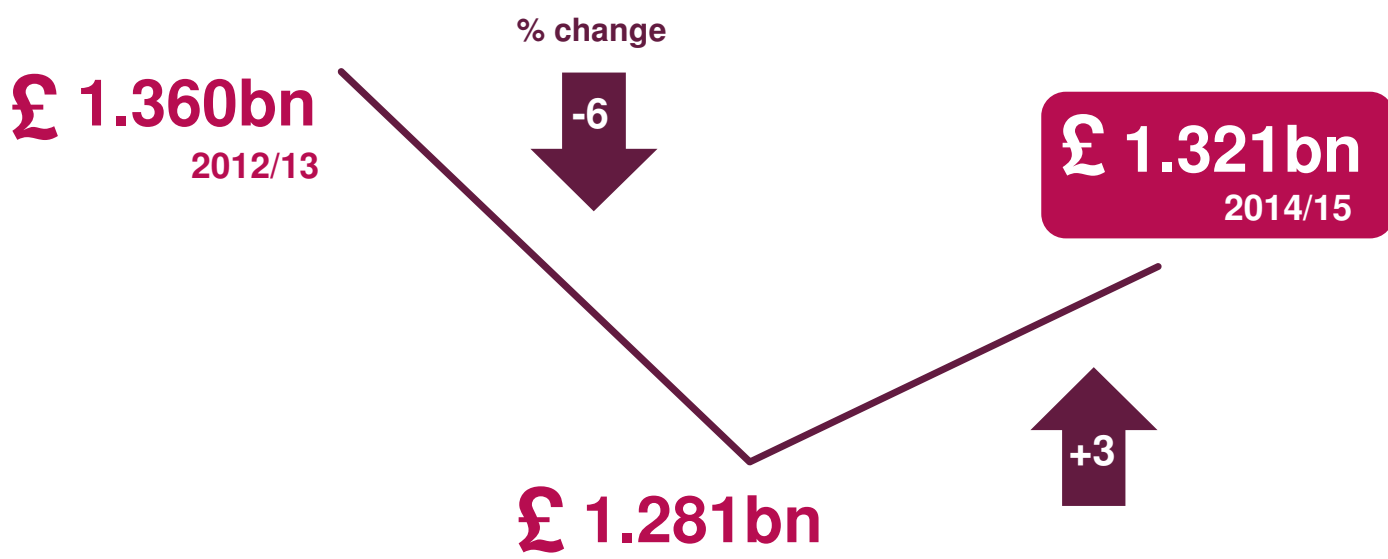

2013/14

Source: Greater Manchester State of the VCSE sector survey 2016/17

Base: 720 All figures are in 2014/15 prices

When the VCSE sector's income is explored in more detail it shows noticeable variations according to organisation size ${ }^{17}$. In $2014 / 15$, the majority of income was concentrated in large and medium sized organisations even though the majority of organisations were micro or small. This is outlined in more detail in figure 5.2.

Figure 5.2: Proportion of organisations and proportion of income by organisation size (2014/15)

Percentage of all organisations

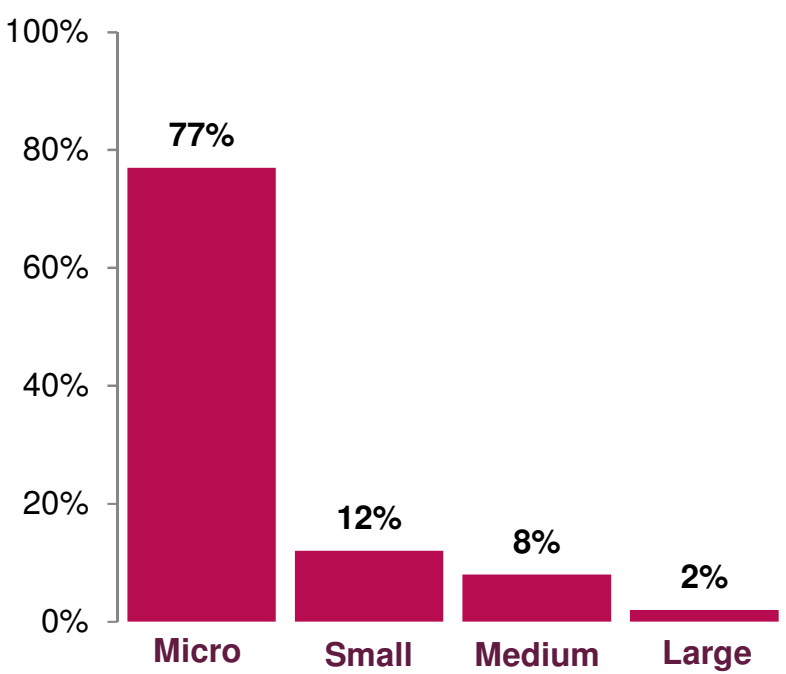

Percentage of all income

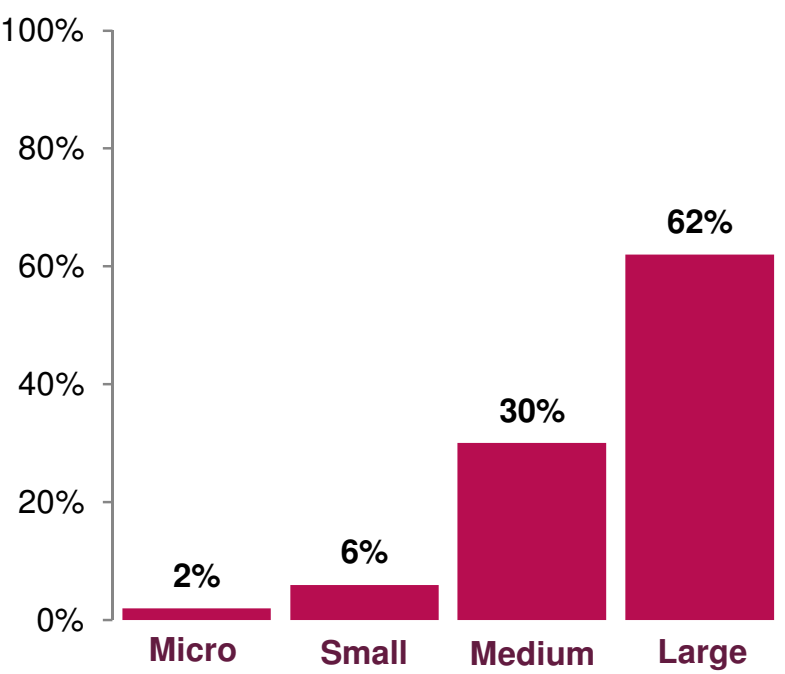

Source: Greater Manchester State of the VCSE sector survey 2016/17

Base: 720

This shows that micro and small organisations account for nine out of ten organisations in the VCSE sector but less than ten per cent of total income. By

\footnotetext{
${ }^{17}$ In exploring organisation size we used the categories developed by NCVO for use in their Almanac series (see e.g. Clark et al., 2010)
} 
contrast medium and large organisations account for just one in ten of the VCSE sector's organisations but receive more than 90 per cent of its income. Income is concentrated particularly in the largest organisations, with around three fifths of all income (62 per cent) into the VCSE sector received by only 374 organisations.

Analysis of income data from survey respondents across Greater Manchester ${ }^{18}$ identified further variations according to organisation size when we explored how income levels had changed between 2012/13 and 2014/15. These are summarised in table 5.1 .

Table 5.1: Estimated change in annual income by organisation size (all Greater Manchester organisations: 2012/13-2014/15)

\begin{tabular}{|c|c|c|c|c|c|c|c|c|}
\hline & \multicolumn{2}{|c|}{$\begin{array}{c}\text { Micro } \\
\text { (under £10k) }\end{array}$} & \multicolumn{2}{|c|}{$\begin{array}{c}\text { Small } \\
(£ 10 k-£ 100 k)\end{array}$} & \multicolumn{2}{|c|}{$\begin{array}{c}\text { Medium } \\
(£ 100 k-£ 1 m)\end{array}$} & \multicolumn{2}{|c|}{$\begin{array}{c}\text { Large } \\
\text { (more than } £ 1 \mathrm{~m} \text { ) }\end{array}$} \\
\hline & Income & $\begin{array}{c}\% \\
\text { change }\end{array}$ & Income & $\begin{array}{c}\% \\
\text { change }\end{array}$ & Income & $\begin{array}{c}\% \\
\text { change }\end{array}$ & Income & $\begin{array}{c}\% \\
\text { change }\end{array}$ \\
\hline 2012/13 & $£ 32.3 \mathrm{~m}$ & & $£ 84.9 \mathrm{~m}$ & & $£ 413.9 \mathrm{~m}$ & & $£ 829.2 \mathrm{~m}$ & \\
\hline $2013 / 14$ & $£ 31.0 \mathrm{~m}$ & -4 & $£ 82.4 \mathrm{~m}$ & -3 & $£ 382.8 \mathrm{~m}$ & -8 & $£ 785.1 \mathrm{~m}$ & -5 \\
\hline $2014 / 15$ & $£ 30.0 \mathrm{~m}$ & -3 & $£ 77.0 \mathrm{~m}$ & -7 & $£ 391.5 \mathrm{~m}$ & 2 & $£ 822.6 \mathrm{~m}$ & 5 \\
\hline
\end{tabular}

Source: Greater Manchester State of the VCSE sector survey 2016/17

Base: 720 All figures are in 2014/15 prices

This shows that across Greater Manchester the micro and small organisation categories experienced year on year reductions in total income between 2012/13 and 2014/15. For micro organisations this is a continuation of a trend identified in the 2012/13 survey where these organisations experienced a reduction of more than 10 per cent between 2010/11 and 2011/12. In contrast the 2012/13 survey identified a small increase in income between 2010/11 and 2011/12 for small organisations.

By contrast medium and large organisations saw a reduction in total income between $2012 / 13$ and 2013/14 but then an increase between 2013/14 and 2014/15. For medium organisations this could indicate the start of a reversal in a trend identified in both the 2010 and 2012/13 surveys where year-on-year reductions in income were identified. This income volatility is a significant challenge in the operating context for medium and large organisations.

\subsection{Sources of Income}

Survey respondents were asked to identify both the public sector bodies from which they received funding in their most recent financial year and any other sources of income (i.e. non-public sector) they received.

Overall, $\mathbf{8 4}$ per cent of respondents received funds from at least one non-public sector source. This is increase of eight percentage points compared to 2012/13. Just over two-thirds (68 per cent) of respondents reported having at least one source of public sector funds. This is higher than the 53 per cent who reported having public sector funds in the 2012/13 survey.

\subsubsection{Other sources of income}

Fundraising was the most frequently identified source of other (i.e. non-public sector) funds (50 per cent of respondents) followed by grants from charitable trusts and

\footnotetext{
${ }^{18}$ It was not possible to undertake sufficiently robust analysis of these trends at a local authority level
} 
foundations (42 per cent). These were also the most common types of funding received in 2012/13.

Fundraising was the most common type of funding selected across all the different areas of Greater Manchester participating in the study apart from the City of Manchester where grants from charitable trusts and foundations was most common.

Respondents were also asked to estimate the proportion of their group or organisation's total income received from each of the non-public sector funding sources. Figure 5.3 shows the most prominent sources of non-public sector funding received and the estimated proportion of total income this represents.

Figure 5.3 shows that for over half (52 per cent) of those receiving income from fundraising, this represented less than 10 per cent of their total income. At the other end of the spectrum for 16 per cent of organisations this represented at least 50 per cent of their total income.

Figure 5.3: Other funds received by Greater Manchester respondents (2014/15)

\section{Fundraising (e.g. crowdfunding events, donations etc.)}

Grants from charitable trusts and foundations

Charging for goods and services

Membership fees I subscriptions

Grants from National Lottery distributors (e.g. BIG)

Business donations or sponsorship

Interest (e.g. bank, endowments, investments)

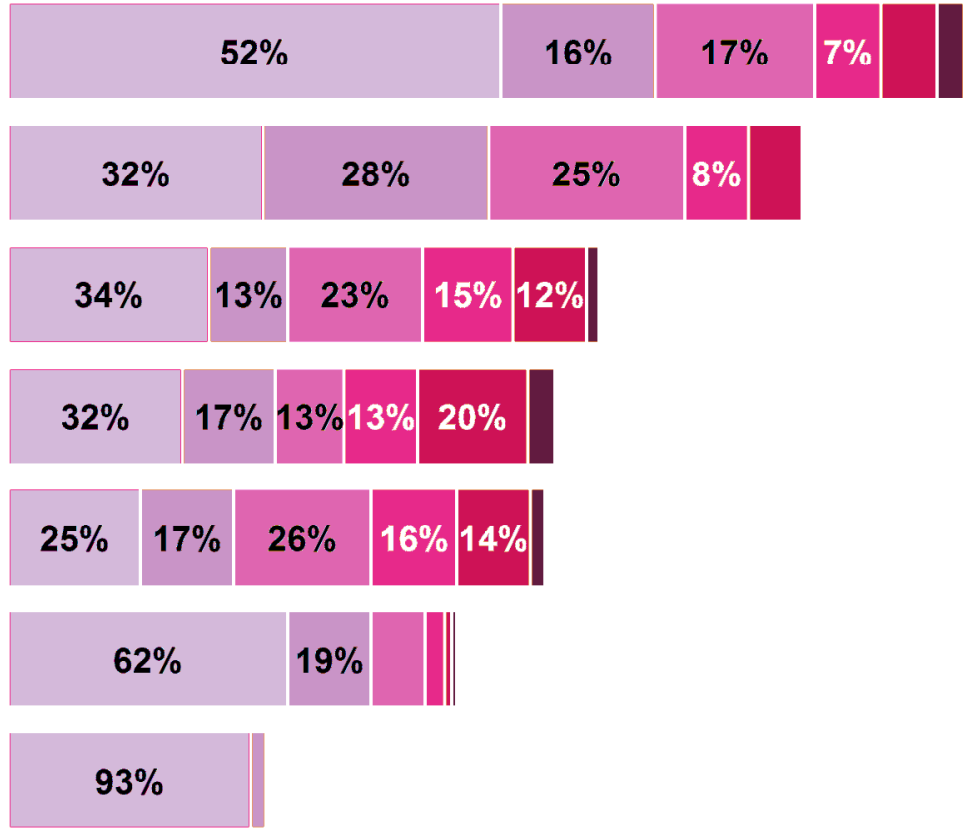

$93 \%$

Less than $10 \%$

At least $10 \%$ but

At least $20 \%$ but

At least $50 \%$ but less than $75 \%$

At least $75 \%$ but less than $100 \%$

$100 \%$

Source: Greater Manchester State of the VCSE sector survey 2016/17

Base: 14-390

Micro organisations were less likely than small, medium and large organisations to have income from non-public sector sources. This is demonstrated by figure 5.4. 
Figure 5.4: Proportion of organisations in receipt of other funds by organisation size (2014/15)

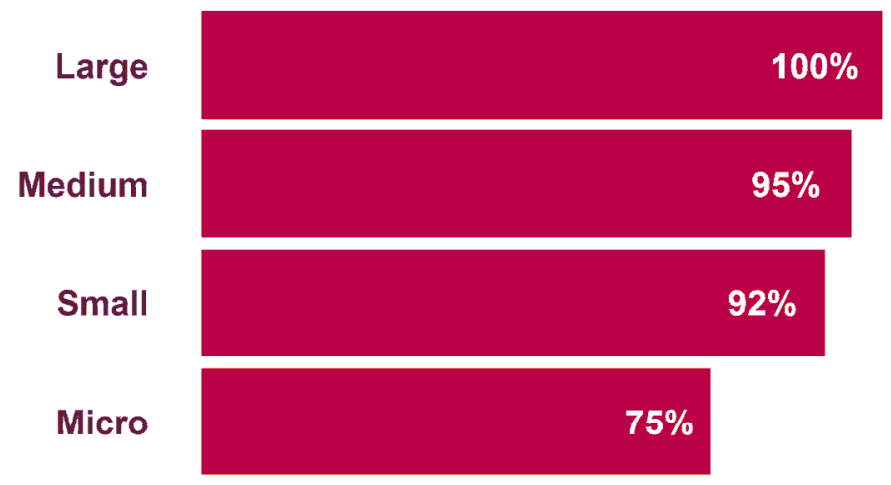

Source: Greater Manchester State of the VCSE sector survey 2016/17

Base: 686

\subsubsection{Public sector income}

Figure 5.5 shows the public sector bodies that survey respondents identified receiving funding from in their most recent financial year.

This shows that local authorities were the most frequently identified source of public sector funding (40 per cent of public sector funding recipients). This was also the most frequently identified source of funding in 2012/13, although the proportion was higher (71 per cent). Almost one fifth (17 per cent) received income from grant schemes administered by local infrastructure organisations followed by 15 per cent of respondents who received income from local public sector health bodies. All other sources received less of a response.

This pattern was reflected across the different areas of Greater Manchester, where local authorities consistently emerged as the most common source of public sector funds. The only exceptions were in Salford; where grant funding administered by Salford CVS on behalf of a public sector body was the most common type of funding; and in Tameside where grant funding administered by Community and Voluntary Action Tameside / Action Together was joint most common with the local authority. 
Figure 5.5: Public sector funds received by Greater Manchester respondents $(2014 / 15)$

Local authorities

Grants administered by local infrastructure organisations

Local public sector health bodies

National government

Greater Manchester Police

Income from Direct Payment or Personal Budgets

Other public sector bodies

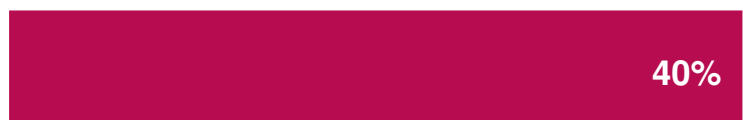

$17 \%$
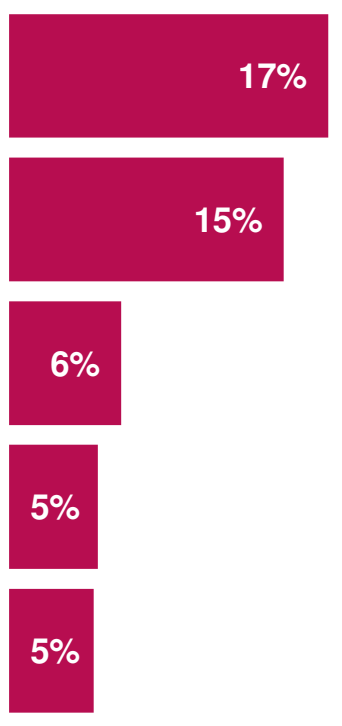

$5 \%$

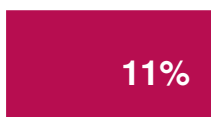

Source: Greater Manchester State of the VCSE sector survey 2016/17

Base: 867

Respondents were also asked to estimate the proportion of their group or organisation's total income each source of public sector income identified represented.

For one quarter (26 per cent) of those receiving funding from local authorities this funding represented less than 10 per cent of their total income. At the other end of the spectrum for 18 per cent of organisations this represented at least 75 per cent of their total income.

For those receiving funding from grants administered by local infrastructure organisations this funding represented less than 10 per cent of their total income for two-fifths $(41 \%)$ of organisations. In contrast for 11 per cent this funding represented at least 75 per cent of income overall.

The survey also revealed notable variations in public sector income received by organisations of different sizes. Micro organisations were less likely than small, medium and large organisations to have at least one source of public sector income. This is outlined in more detail in figure 5.6.

This shows that only 54 per cent of micro organisations that responded to the survey received public sector funding compared to 75 per cent of small organisations, 85 per cent of medium organisations and 96 per cent of large organisations. 
Figure 5.6: Proportion of Greater Manchester organisations in receipt of public sector funds by organisation size (2014/15)

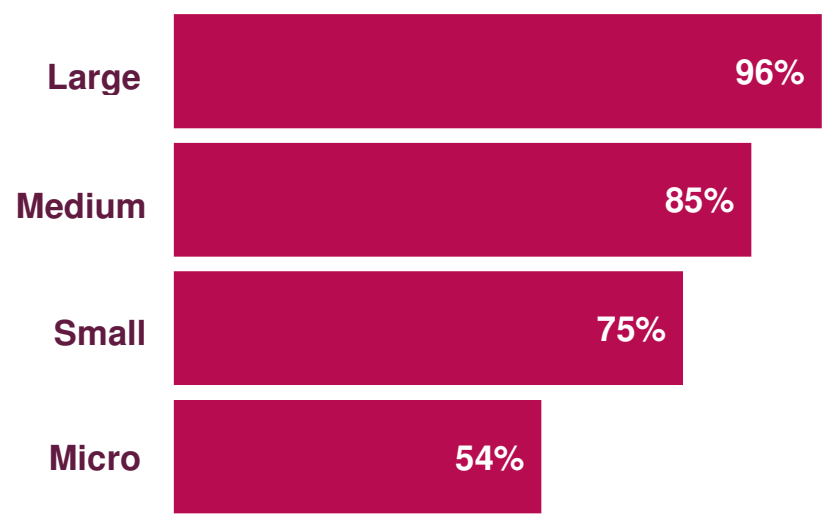

Greater Manchester State of the VCSE sector survey 2016/17 Base: 686

\subsection{Financial Sustainability}

The survey asked respondents about how their organisation's financial situation had changed in the past 12 months (i.e. during the current financial year). The results are outlined in figure 5.7 .

Figure 5.7: Change in financial circumstances in the last 12 months

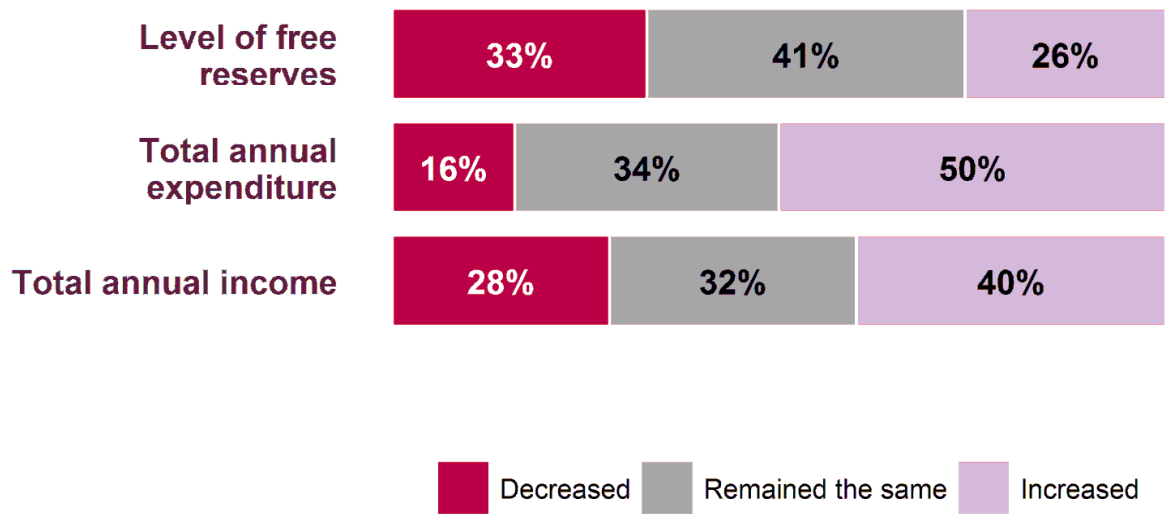

Source: Greater Manchester State of the VCSE sector survey 2016/17

Base: 784 (income), 767 (expenditure), 696 (free reserves)

Note: 'cannot say' response has been excluded from the analysis

This raises some concerns: 50 per cent of respondents reported increasing their expenditure but only 40 per cent had experienced an increase in income and only 26 per cent reported an increase in reserves. In addition, 28 per cent of respondents reported a decrease in income but only 16 per cent reduced their expenditure.

23 per cent of respondents provided an expenditure figure for 2014/15 that was greater than their income. This means that there were a notable number of organisations that spent more money than they received in the past $\mathbf{1 2}$ months. This figure is, however, lower than the 34 per cent of organisations who provided an expenditure figure in the 2013 study that was greater than their income, nevertheless it still appears that the sustainability of a sizeable number of organisations could be at risk. 
Explored by organisation size, the data suggests that reductions in income were more common in medium and large organisations than smaller ones. In terms of expenditure, large organisations were more likely to report an increase. This is outlined in more detail for all sizes of organisations in figures $5.8 \mathrm{a}$ and $5.8 \mathrm{~b}$ below.

Figure 5.8a: Change in income in the last 12 months by organisation size

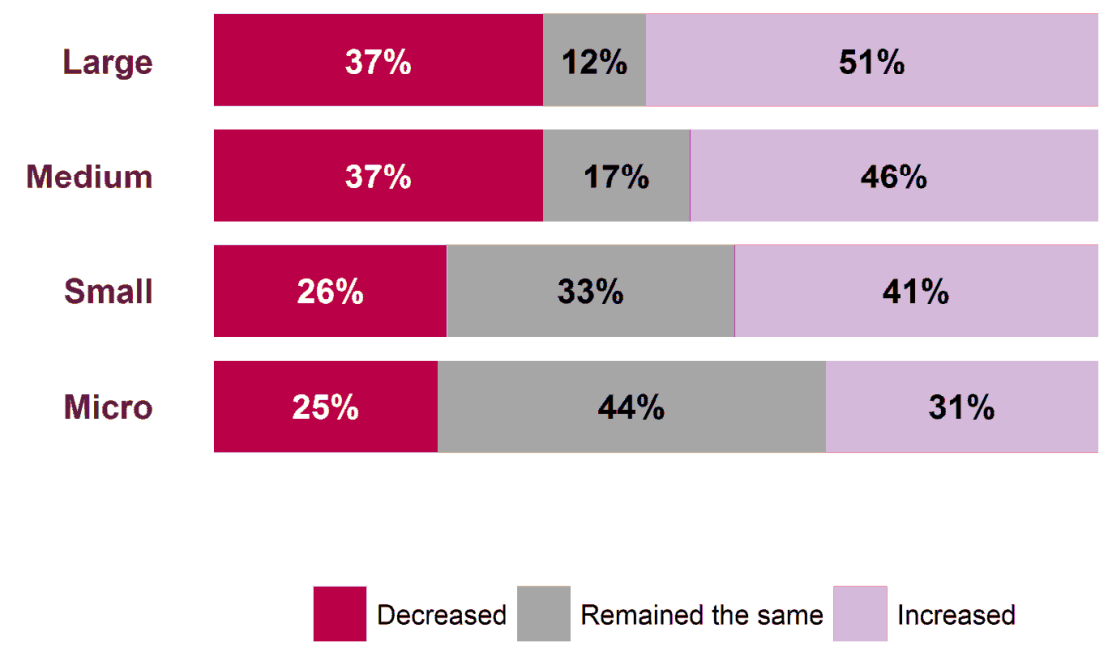

Source: Greater Manchester State of the VCSE sector survey 2016/17

Base: 661

Note: 'cannot say' response has been excluded from the analysis

Figure 5.8b: Change in expenditure in the last 12 months by organisation size

\begin{tabular}{|c|c|c|c|}
\hline Large & $28 \%$ & $9 \%$ & $63 \%$ \\
\hline Medium & $24 \%$ & $19 \%$ & $56 \%$ \\
\hline Small & $15 \%$ & $33 \%$ & $52 \%$ \\
\hline Micro & $10 \%$ & $45 \%$ & $44 \%$ \\
\hline & & ased & Remained the same \\
\hline
\end{tabular}

Source: Greater Manchester State of the VCSE sector survey 2016/17

Base: 651

Note: 'cannot say' response has been excluded from the analysis

Further analysis of the financial reserve levels reported by respondent organisations provides an additional insight in to the financial health of the VCSE sector. Reserves are important as they provide organisations with funds to fall back on in the short term should other sources of funding reduce or be withdrawn. They also provide organisations with the flexibility to develop new and innovative activity that might not have attracted external funding from the outset. Organisations with low reserves relative to expenditure are therefore more likely to be restricted in their ability to adapt if key external funding is lost. In order to explore this issue in more detail 
reserves (2014/15) were calculated as a proportion of expenditure $(2014 / 15)$ for each respondent. The results are shown in figure 5.9.

Figure 5.9: Financial vulnerability of organisations in Greater Manchester

Less than 1 month expenditure in reserve

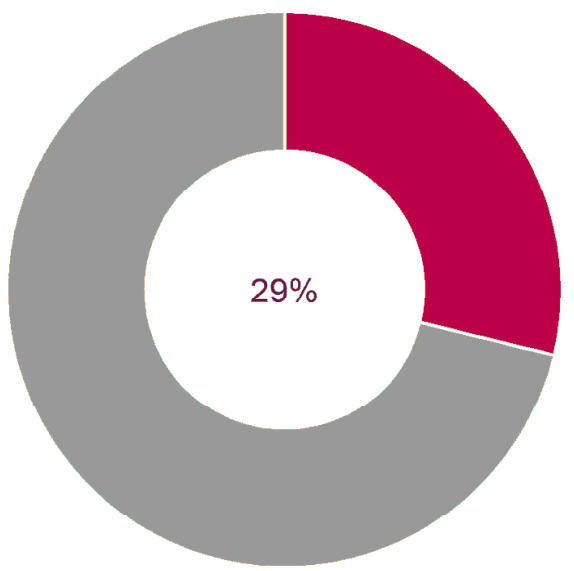

Less than 3 months of expenditure in reserve

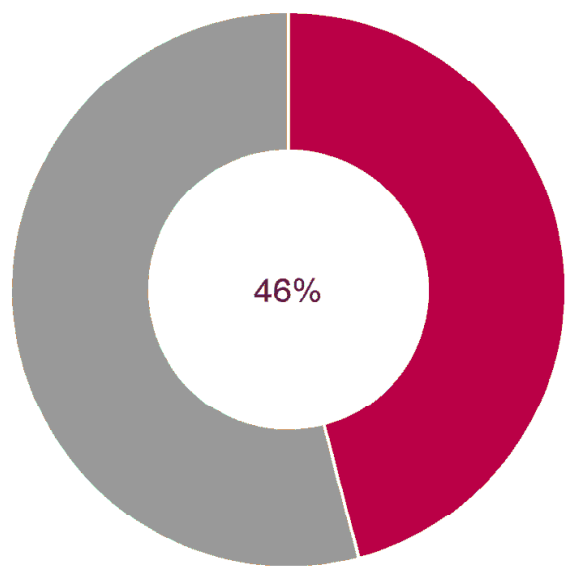

Source: Greater Manchester State of the VCSE sector survey 2016/17

Base: 649

This shows that 29 per cent had reserve levels of less than one month's expenditure, and a further 17 per cent had reserves that covered less than three month's expenditure. This suggests that over two-fifths of all organisations in the VCSE sector could be vulnerable should their funds reduce severely. Results are similar to the 2012/13 survey when 41 per cent of organisations reported reserves equalling less than three month's expenditure (although just 15 per cent of organisations had reserve levels of less than one month's expenditure in 2012/13).

Survey respondents were also asked how they thought the environment for funding/income for the VCSE sector will change over the next year. Figure 5.10 shows the responses received to this question. This shows that over half (56 per cent) of organisations in Greater Manchester thought the environment will deteriorate compared to just seven per cent who felt the environment is set to improve. 14 per cent saw the environment for funding/income staying the same. Analysis across the seven study areas finds a similar pattern, although respondents were more positive in Salford where 12 per cent felt the environment is set to improve compared to just eight per cent or fewer in the other six areas. 
Figure 5.10: Change in the environment for funding/income in the next year

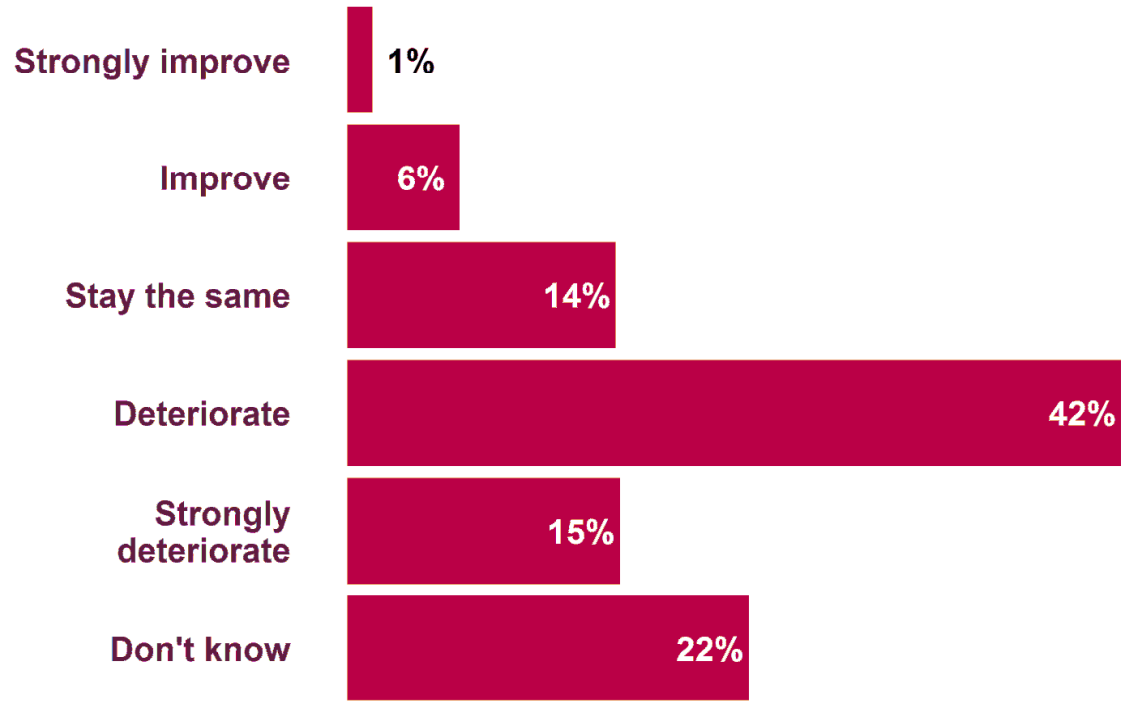

Source: Greater Manchester State of the VCSE sector survey 2016/17

Base: 891 


\section{Paid Employees}

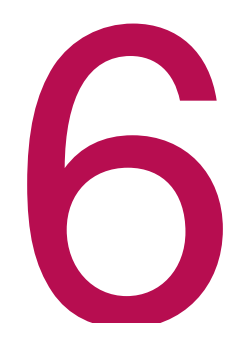

This chapter looks at the paid workforce of the VCSE sector in Greater Manchester.

\subsection{How many FTE paid staff are employed in the VCSE sector in Greater Manchester?}

Based on the average number of FTE paid staff employed by organisations responding to the survey across Greater Manchester, and drawing on the assumptions used to estimate the total number of organisations in Greater Manchester, it is estimated that:

\section{8,600 FTE paid staff were employed in the VCSE sector in Greater Manchester in 2016/17}

This represents 42,600 employees.

This is a higher figure than the 23,600 FTE paid staff estimated to work in the sector in the 2013 study.

Gross Value Added (GVA), the value of goods and services produced, is a key measure of the economic contribution of organisations or VCSE sectors. It can be estimated for paid staff working in Greater Manchester organisations by multiplying the number of FTE paid staff by the estimated gross value added (GVA) per FTE employee ${ }^{19}$. From this calculation it is estimated:

\section{£905.3m contributed to the economy per annum by paid staff of Greater Manchester VCSE sector organisations}

Figure 6.1 presents a breakdown of responding organisations by the number of FTE paid staff they employed. Just over three-quarters (77 per cent) of organisations employed less than five FTE paid staff members. Included in this figure were 51 per cent of organisations that did not employ any paid staff. Further analysis reveals that the majority of these were micro organisations with income of less than $£ 10,000$. At the other end of the spectrum ten per cent of organisations employed 20 or more FTE paid members of staff. This pattern is broadly equivalent to that identified in the 2012/13 survey, although the proportion of organisations with no staff was higher at 64 per cent.

\footnotetext{
${ }^{19}$ This study used Greater Manchester GVA per employee averaged across the following two VCSE sectors: education and human health and social work activities.
} 
Figure 6.1: Organisations by numbers of FTE paid staff

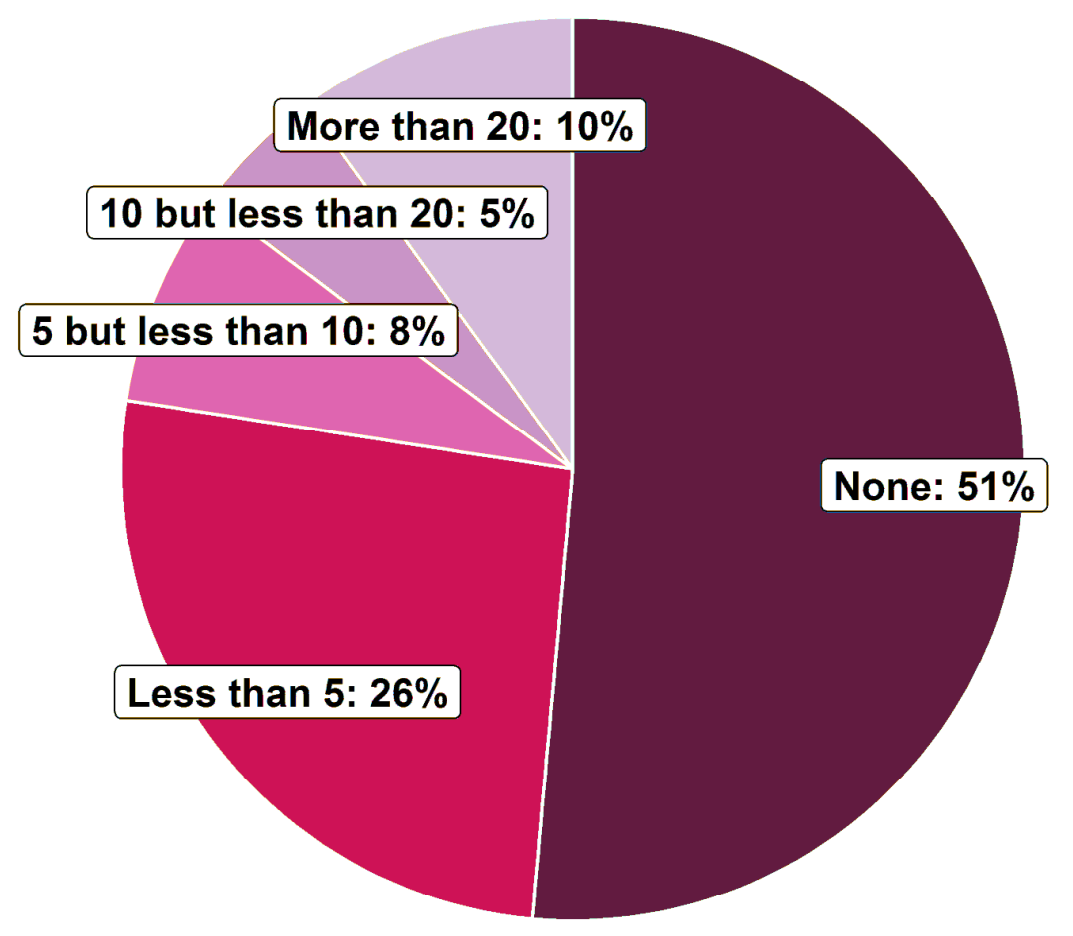

Source: Greater Manchester State of the VCSE sector survey 2016/17 Base: 1,015

\subsection{How has the VCSE sector's workforce changed in the last 12 months?}

The survey asked respondents whether the number of staff in their organisation's workforce had 'increased', 'remained the same' or 'decreased' this year compared to the previous year. Figure 6.2 presents the results to this question, the key findings of which are:

\section{Paid staff:}

- 61 per cent of organisations employed a similar number of paid staff compared to a year ago

- 22 per cent of organisations reported an increase in paid staff compared to 16 per cent that reported a decrease

- $\quad$ an equivalent percentage of organisations reporting a change in 2012/13 reported an increase in paid staff as a decrease (17 per cent). 
Figure 6.2: Change in aspects of the workforce (paid staff) in the last 12 months

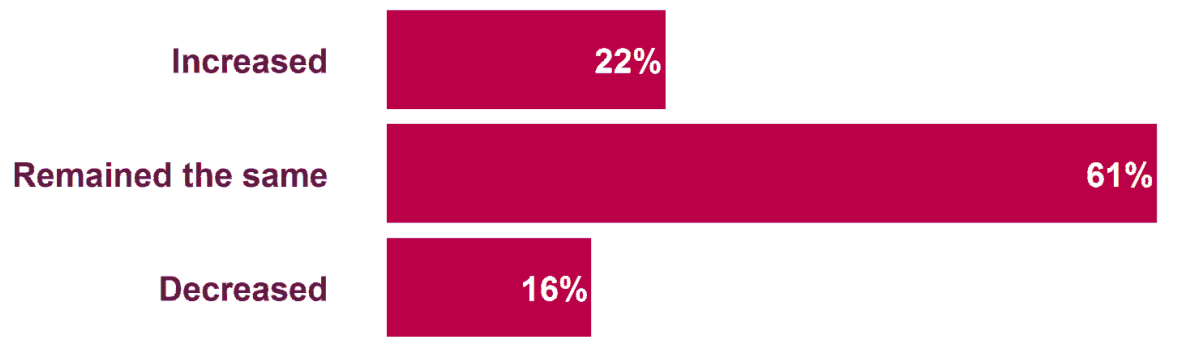

Source: Greater Manchester State of the VCSE sector survey 2016/17

Base: 887

Note: 'cannot say' response has been excluded from the analysis 


\section{Volunteers}

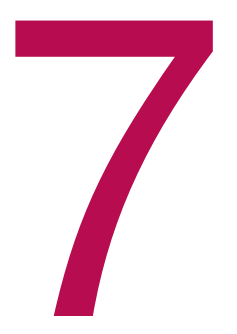

This chapter looks at the volunteers within the VCSE sector in Greater Manchester.

7.1. How many volunteers are part of the VCSE sector workforce in Greater Manchester and what is their economic contribution?

Based on responses to the survey across Greater Manchester on questions exploring the numbers of volunteers and committee/board members and the hours which they contribute, and drawing on the assumptions used to estimate the total number of organisations in Greater Manchester, it is estimated there are:

\section{1,800 volunteers in the VCSE sector's workforce in Greater Manchester in 2016/17}

This includes:

\section{5,800 volunteers in the VCSE sector's workforce in Greater Manchester in 2016/17}

\section{5,900 committee/board members in the VCSE sector's workforce in Greater Manchester in 2016/17}

This figure for volunteers is equivalent to 13 per cent of Greater Manchester's total population $(2,756,200)$.

It is also estimated that:

\section{1 million hours of their time is provided by these volunteers and committee/board members per week}

The 2012/13 study estimated there were 334,200 volunteers in Greater Manchester who provided 1.1 million hours per week. The previous study did not ask for volunteers and committee/board members to be recorded separately so caution should be applied when making comparisons.

\footnotetext{
${ }^{20}$ It is possible that some of these volunteers could be the same person volunteering for multiple organisations.
} 
There are two broad approaches to valuing the contribution of volunteers. One method, and this study's preferred approach, is to value the output that they produce. In effect this is the value to society of the goods and services that volunteers produce. This can be estimated by multiplying the number of FTE volunteers by the estimated gross value added (GVA) per FTE employee. ${ }^{21}$

From this calculation:

\section{$£ 973.0$ million per annum estimated as the economic contribution of volunteers and committee/ board members in Greater Manchester organisations}

The use of estimated GVA per FTE employee to measure the value of the output produced by volunteers assumes that paid employees would not be used in the absence of volunteers to produce the same level of goods and services. In such a situation the value of output is the value of the labour input (wages and benefits) plus the value of the capital input (for example office space and computers). If paid employees would be used to produce the same level of goods and services then the value of capital input would be borne whether or not volunteers were used. Therefore the value of the output from volunteers would be just the value of the labour input. This value would be roughly equivalent to the value estimated from the input method of valuation which is outlined in the next paragraph.

In the second method, the value of the input of volunteers is used to value the contribution of volunteers ${ }^{22}$. This is the amount that it would cost to pay employees to do the work carried out by volunteers. As such, this can be considered to be the benefit to organisations ${ }^{23}$. However, this benefit might also be passed onto society via lower prices for goods and services due to lower costs of production. The input value of volunteers can be calculated by multiplying the number of hours that volunteers give per week by an estimate of how much it would cost to employ someone to do that work. There are a number of widely accepted hourly rates that could be used to estimate this value; these include: the national minimum wage or national living wage, the local median wage, the local mean wage and the reservation wage. The preference in this study has been to provide a range using the national living wage (low estimate) and the local median wage (high estimate). In reality the true value of the input provided by volunteers will lie between the two estimates. It is estimated that:

- $\quad$ assuming the national living wage for adults ${ }^{24}$ it would cost $\$ 402.6$ million annually to employ staff to do the work provided by volunteers in Greater Manchester organisations

- $\quad$ assuming the median gross hourly wage for full time employees in Greater Manchester ${ }^{25}$ it would cost $£ 719.0$ million annually to employ staff to do the work provided by volunteers in Greater Manchester organisations.

Figure 7.1 presents a breakdown of responding organisations by the number of volunteers that they use. Five per cent of respondents indicated they had no volunteers. This is in contrast to the 2012/13 survey when just one per cent of

\footnotetext{
${ }^{21}$ This study used Greater Manchester GVA per employee averaged across the following two VCSE sectors: education and human health and social work activities.

22 This is the approach recommended by Volunteering England

${ }^{23}$ This assumes that there are no additional costs faced by organisations in using volunteers: for example extra management costs

${ }^{24} £ 7.20$ for 25 years and older in 2016

$25 £ 12.86$ for 2016
} 
organisations reported having no volunteers. Just under one fifth (18 per cent) of respondents to the latest survey had 50 or more volunteers, an increase since 2012/13 when this figure was 14 per cent.

This pattern was fairly similar within each of the seven Greater Manchester areas that were part of the detailed study.

Figure 7.1: Organisations by numbers of volunteers

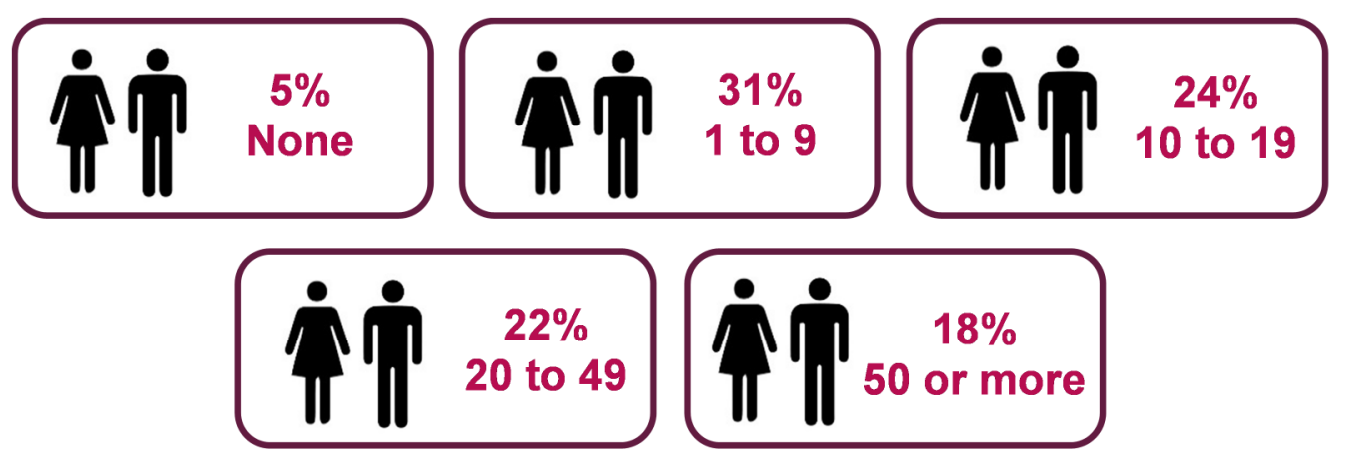

Source: Greater Manchester State of the VCSE sector survey 2016/17

Base: 1,111

\subsection{How has the VCSE sector's workforce changed in the last 12 months?}

The survey asked respondents whether the number of volunteers in their organisation's workforce had 'increased', 'remained the same' or 'decreased' this year compared to the previous year. Figure 7.2 presents the results to this question, the key findings of which are:

- 42 per cent of respondents reported increased numbers of volunteers now compared to a year ago

- in comparison 13 per cent of organisations reported a decrease in volunteer numbers.

The 2012/13 survey found similar results:

- 39 per cent of respondents reported increased volunteer numbers

- $\quad$ half (50 per cent) reported that volunteer numbers remained the same

- $\quad$ while 11 per cent reported that numbers of volunteers decreased. 
Figure 7.2: Change in aspects of the workforce (volunteers) in the last 12 months

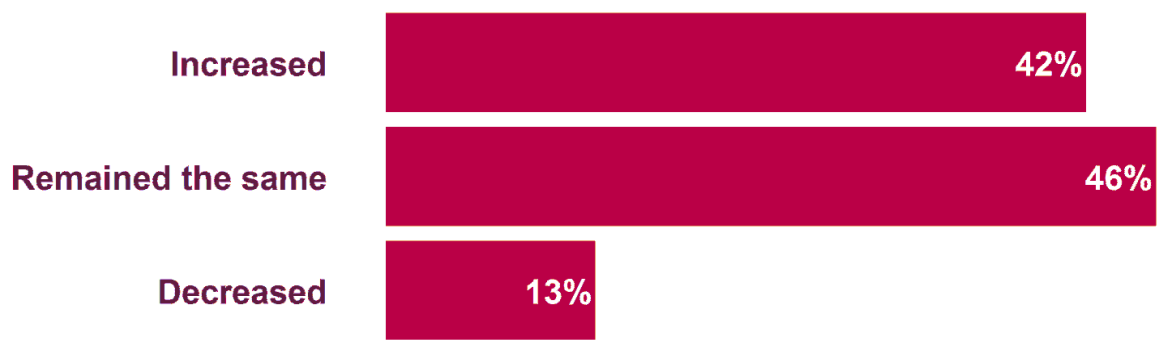

Source: Greater Manchester State of the VCSE sector survey 2016/17

Base: 1,040

Note: 'cannot say' response has been excluded from the analysis

\subsection{Qualitative responses on volunteering}

\section{The benefits of volunteering}

Focus group participants from across Greater Manchester highlighted volunteering as an essential and highly valuable feature of the 'identity' of the VCSE sector and its work supporting people and communities. A range of benefits were consistently identified with volunteering:

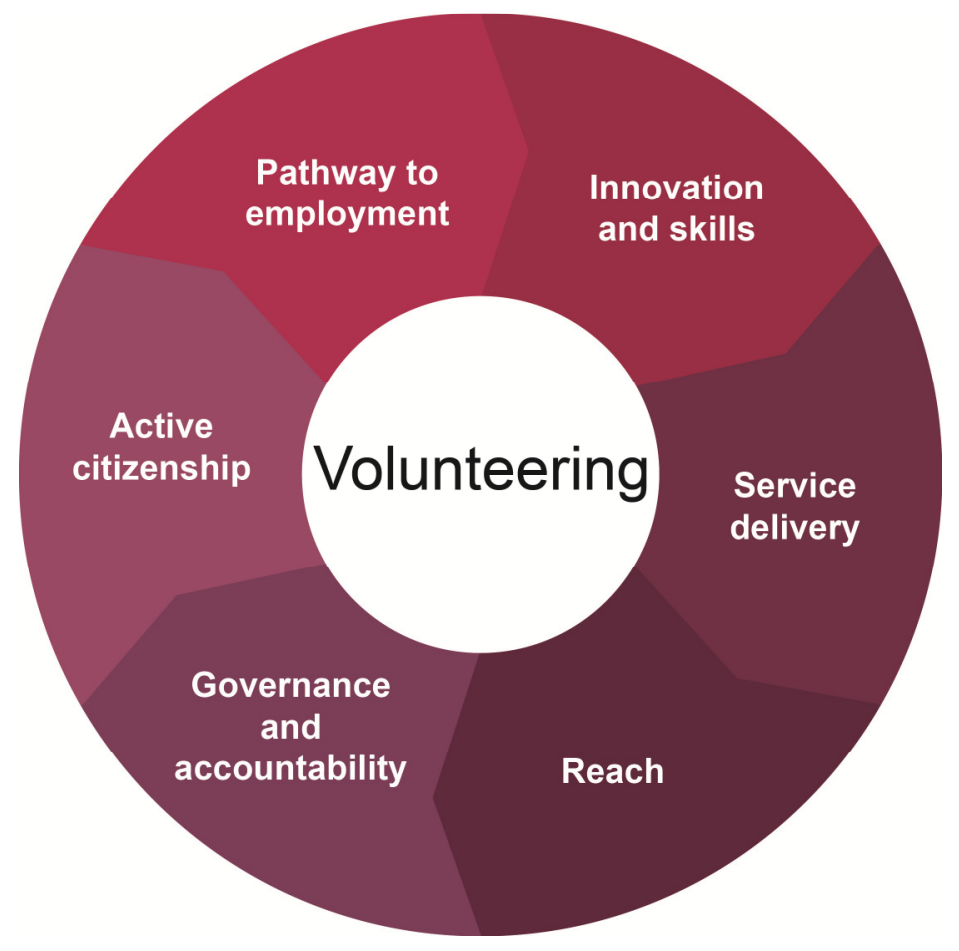

- Innovation and skills: bringing new ideas, experiences, perspectives and expertise to VCSE organisations.

- Service delivery: enabling VCSE organisations to maintain and improve existing services or develop new ones. 
- Reach: enabling organisations to support parts of the community which otherwise could not be reached to enter new fields of activity, and to better understand service-users with whom they are already working.

- Governance and accountability: improving staff accountability and responsibility, bringing their broad experiences and 'grounded' perspective to VCSE organisations' work and revitalising internal democratic processes.

- $\quad$ Active citizenship: acting as role models within VCSE organisations, both for service-users and the wider community, including promoting the work of VCSE organisations prompting more people to become involved.

- Pathway to employment: using volunteering as a springboard to new careers, often in health, social care, social work, education and community-development fields.

\section{Changes in volunteering}

The focus groups provided a mixed picture of whether or not levels of volunteering had changed in recent years. Participants in a number of areas reported an increase in the number of volunteers whilst in other areas this was said to have remained relatively stable.

"We see more people coming in and seeking opportunities from a variety of backgrounds. The 'drivers' are similar, but we just see more people coming through."

"We use many volunteers. But I don't think there is a significant increase or decrease. Once we get them, we do keep them."

In general, larger VCSE organisations were more likely to have reported an increase in volunteers whilst smaller organisations were more likely to be struggling to retain and recruit volunteers.

Where volunteering levels had increased this was equated in part to an increase in the number of young people seeking to volunteer.

"We have a lot more students now and we can help them focus on the jobs they want."

It was reported that young people increasingly saw volunteering as 'stepping stone' to a professional career, gaining valuable work experience to enhance their CVs.

\section{Challenges associated with volunteering}

Focus group participants identified a number of challenges associated with volunteering. Foremost amongst these was the need for 'quality volunteers'. VCSE organisations either needed dedicated and skilled volunteers with knowledge and experience of the type of work being undertaken, or they needed sufficient resources to support recruit, train and co-ordinate volunteers on a consistent basis. In a climate of limited funding opportunities from the public sector, the processes of development and maintaining a suitable volunteer 'workforce' are increasingly challenging.

"Volunteers are invaluable for what we do and what we are, but the time and resources needed to manage all that and get something out of it, can be exhausting."

The focus groups highlighted differences in the intensity and extent of these challenges and the effect they have for different types of VCSE organisation. Smaller 
organisations seemed to be most acutely affected due to their more limited resources and personnel, as well as their higher dependence on volunteering for continuing to offer their services against a backdrop of increasing demand. Many of these smaller organisations faced difficulties when 'competing' for grants and contracts, which expect certain standards and policies with respect to volunteers that they do not have the capacity to implement.

"Of course, there is a cost to think of. An organisation of our size... who pays for their DBS? We do. Who pays for their training certificates to get them to level 1? We do. Who pays for my wage? Well, somebody's got to. There is a cost element to it but the argument is, when you go out for bids, $10 \%$ of your marks is the added value you can bring in from volunteers." 


\section{Partnership Working: the Public Sector}

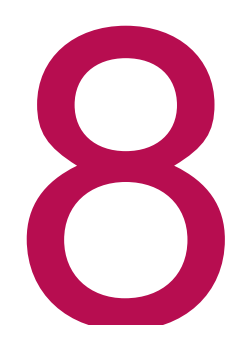

This chapter considers the relationship between the VCSE sector and the public sector, exploring organisation's experiences of partnership working with public sector bodies.

\section{1. $\quad$ Dealings with local public sector bodies}

Survey respondents were asked about the extent of their dealings with each of the local authorities in Greater Manchester.

Overall, 84 per cent of respondents in Greater Manchester had some dealings with at least one local authority (an increase from 72 per cent in 2012/13): 16 per cent had a great amount of dealings with at least one local authority and 36 per cent had a fair amount of dealings.

As figure 8.1 shows there was some variation across local area.

Figure 8.1: Dealings with Greater Manchester local authorities

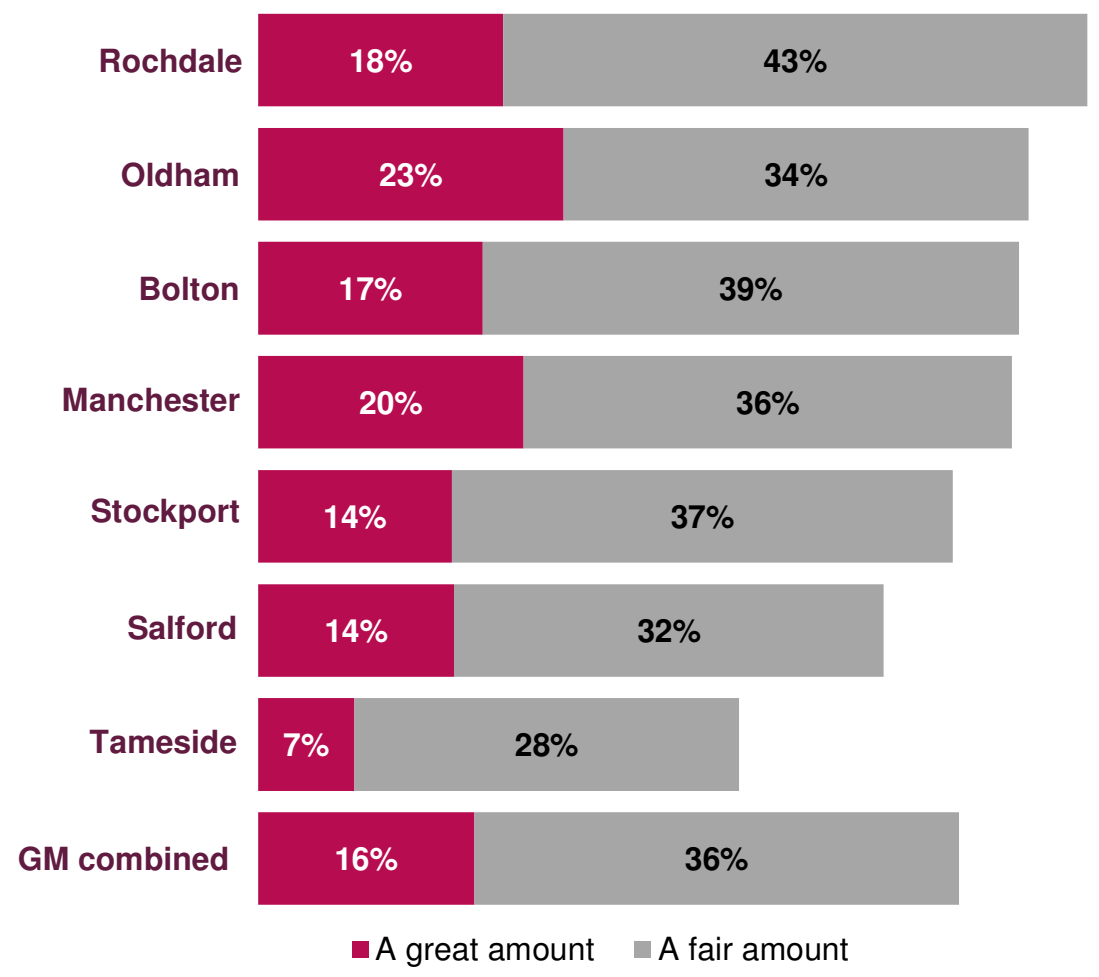

Source: Greater Manchester State of the VCSE sector survey 2016/17

Base: 1,080 
Respondents were also asked to consider the extent to which their organisation has direct dealings with any emerging Greater Manchester structures (e.g. Greater Manchester Combined Authority, The Office of the Police and Crime Commissioner, The Health and Social Care Devolution Team etc.). Figure 8.2 presents the results to this question.

Just under two-fifths (38 per cent) had some dealings with any of the emerging structures including just two per cent who had a 'great amount' of dealings and ten per cent who had a 'fair amount' of dealings. This pattern was broadly similar across the seven Greater Manchester areas.

Figure 8.2: Dealings with emerging Greater Manchester structures

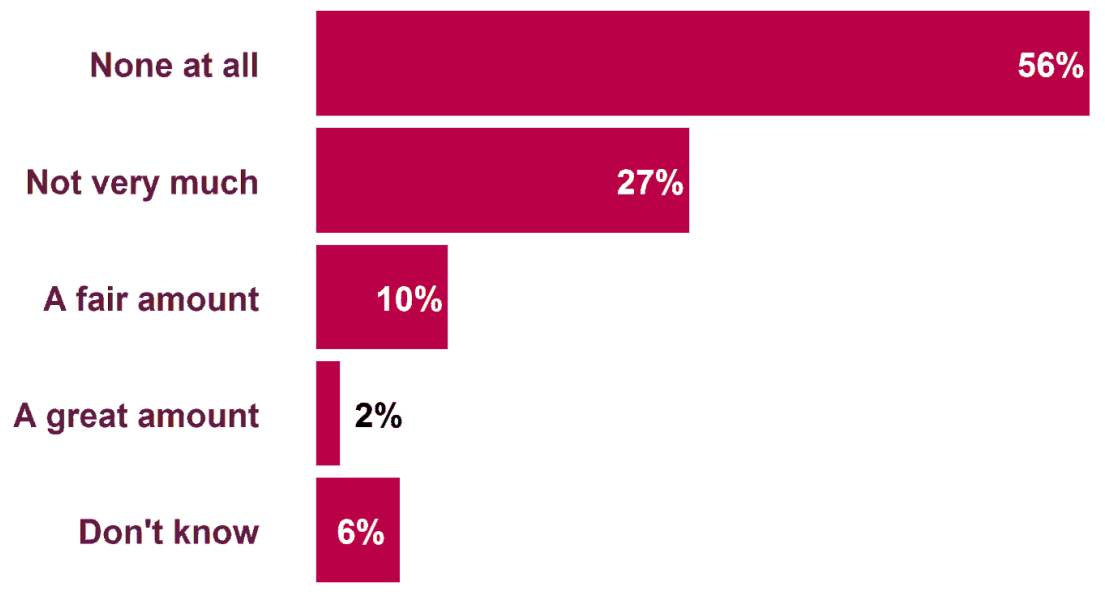

Source: Greater Manchester State of the VCSE sector survey 2016/17

Base: 977

\subsection{Relationships with local public sector bodies}

Survey respondents were also asked two further questions about the extent to which their organisations were satisfied with their ability to influence public sector decisions of relevance to their organisation and the extent to which they thought local statutory bodies influenced their success ${ }^{26}$. The results of these questions are summarised in figure 8.3.

Figure 8.3 shows that 30 per cent of respondents were satisfied with their ability to influence local authorities' decisions of relevance to their organisation and 38 per cent said local authorities had a positive influence on their organisation's success. Results are similar to 2012/13 when 28 per cent of respondents were satisfied with their ability to influence local authorities' decisions of relevance to their organisation and 39 per cent said that local authorities had a positive influence on their organisation's success.

In addition, 36 per cent of respondents said they were satisfied with their ability to influence the key decisions of their most frequent other public sector contact and 51 per cent said this contact had a positive influence on their success. These responses are more positive than in 2012/13 when the figures were 23 per cent and 37 per cent respectively.

\footnotetext{
${ }^{26}$ This latter measure was used in 2008 and 2010 to provide evidence of local authority performance against 'National indicator 7: the environment for a thriving third sector'.
} 
Figure 8.3: Proportion of organisations who said they were satisfied with their ability to influence public sector decisions of relevance to their organisation and who said local public sector bodies influence their organisation's success

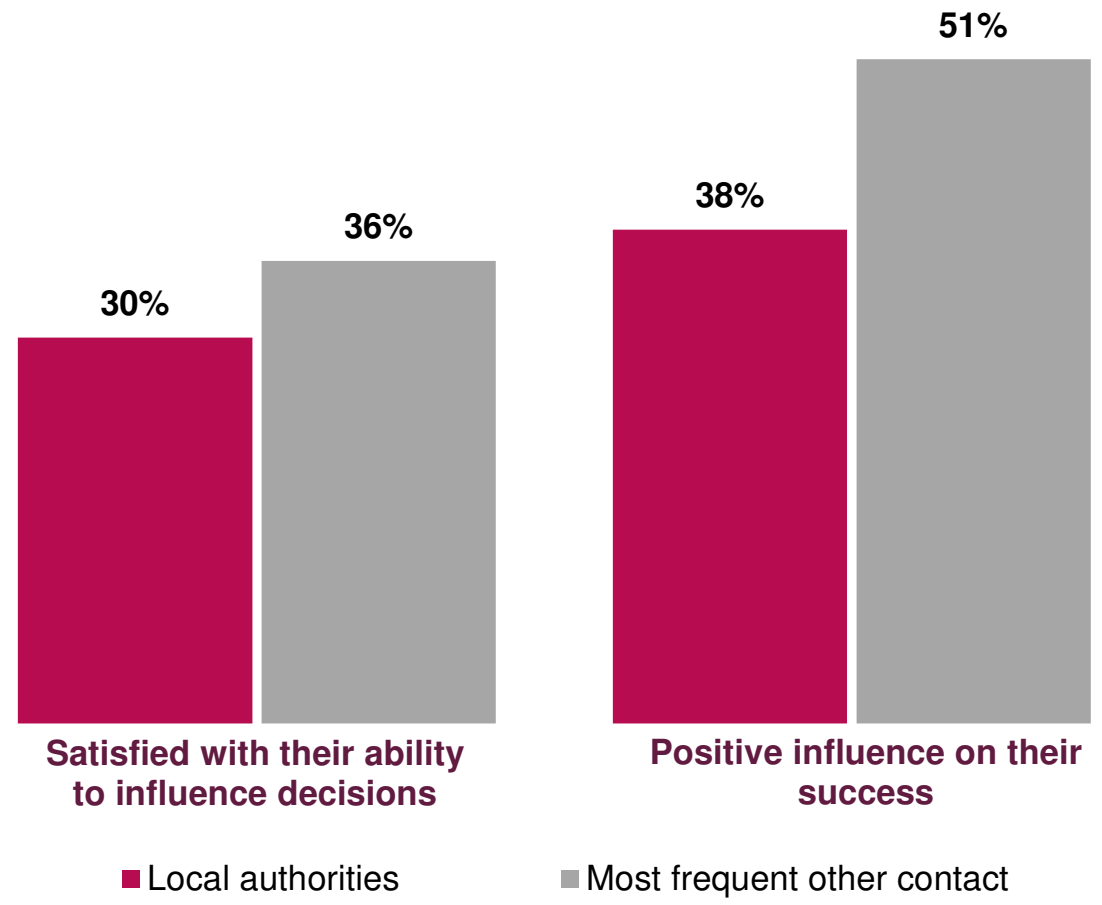

Source: Greater Manchester State of the VCSE sector survey 2016/17

Base: 897/570 (ability to influence), 889/605 (positive influence)

\subsection{Funding from local public sector bodies}

Respondents were also asked to reflect on their experiences of public sector funding in terms of how successful they had been, how satisfied they were with bidding arrangements, and how satisfied they were with the level of opportunity to bid for long-term funding.

Figure 8.4 shows responses to the question which asked organisations to consider how successful they had been in applying for funding or bidding for contracts.

This shows that 45 per cent of respondents were successful in bidding for funding or contracts with local authorities compared to a 40 per cent success-rate with other public sector bodies. In 2012/13 a higher proportion indicated they had been successful in bidding for funding or contracts from local authorities (50 per cent) but the figure for other public sector bodies was lower (34 per cent). 
Figure 8.4: Success bidding for funding and contracts

$45 \%$

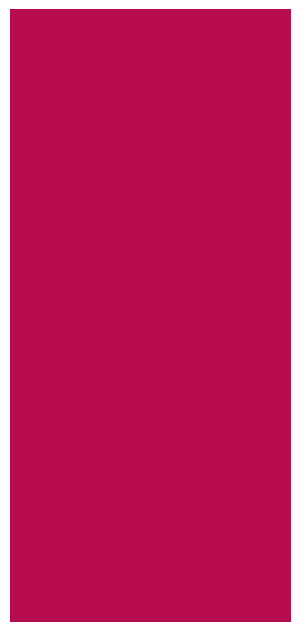

$40 \%$

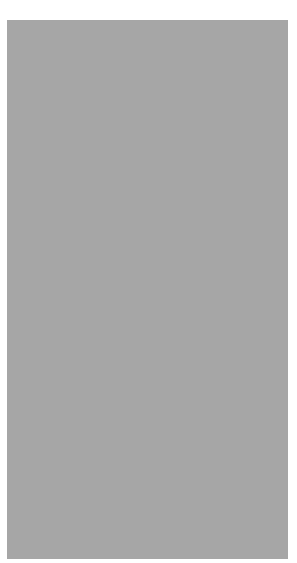

Successful in bidding for funding/contracts

- Local authorities

- Other public sector bodies combined

Source: Greater Manchester State of the VCSE sector survey 2016/17 Base: 1,060/1,036

Respondents were asked specifically about local authorities and how satisfied they were with their grant funding and contract bidding arrangements and opportunities for funding and contracts lasting three years or longer. The responses are illustrated in figure 8.5 .

Two-fifths (40 per cent) were satisfied with grant funding arrangements. Satisfaction with contract bidding arrangements was lower at 25 per cent. Satisfaction with opportunities for both funding and contracts lasting three years or longer was lower (both below 20 per cent). This pattern was broadly similar across the seven Greater Manchester areas.

In 2012/13 respondents were not asked separately about grant funding and contracts. Almost half (48 per cent) of respondents were satisfied with local authorities' funding/bidding arrangements in 2012/13 and 23 per cent were satisfied with their opportunities for funding/contracts which lasted three years or longer. 
Figure 8.5: Experiences of bidding for funding and contracts with local authorities

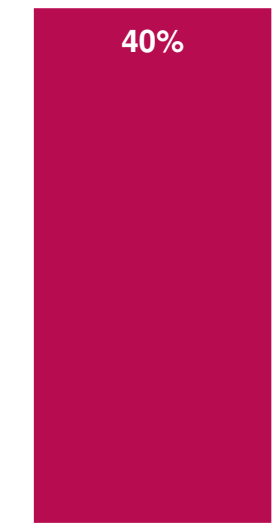

Satisfied with grant funding arrangements

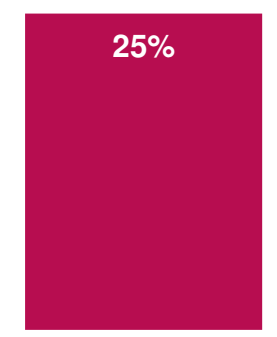

Satisfied with contract bidding arrangements

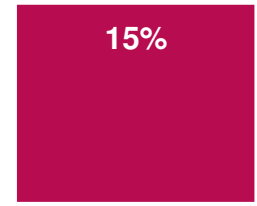

Satisfied with opportunities for

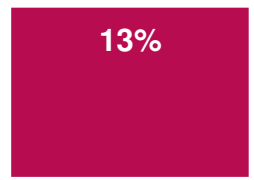

Satisfied with opportunities for funding that lasts 3yrs+ contracts that last 3yrs+

Source: Greater Manchester State of the VCSE sector survey 2016/17

Base: 808 (grant funding arrangements), 631 (contract bidding arrangements), 703 (opportunities for funding), 605 (opportunities for contracts)

Survey respondents were asked to consider how satisfied they were with the grant funding and contract bidding arrangements of their most frequent other public sector contact. As figure 8.6 shows, over one quarter ( 25 per cent) indicated they were satisfied.

They were also asked about their satisfaction with opportunities for funding and contracts longer than three years. Just 11 per cent were satisfied.

Comparing responses across the seven Greater Manchester areas found satisfaction with grant funding and contract bidding arrangements was higher than satisfaction with opportunities for funding and contracts longer than three years in all areas.

Figure 8.6: Experiences of bidding for funding and contracts with other public sector bodies

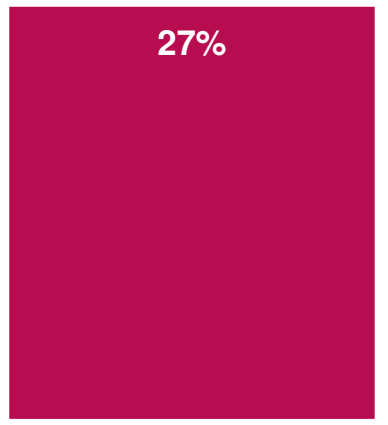

Satisfied with grant funding / contract bidding arrangements

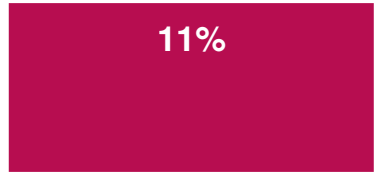

Satisfied with opportunities for funding / contracts that last 3yrs+

Source: Greater Manchester State of the VCSE sector survey 2016/17

Base: 705 (funding/bidding arrangements), 687 (opportunities for funding/contracts); 


\subsection{Qualitative responses on relationships between the VCSE sector and local public sector bodies}

\section{Devolution}

Much focus group discussion focussed on the prospects for devolution in Greater Manchester and its potential impact on VCSE organisations in each area. Overall, participants expressed uncertainty about the implications of devolution for their work and many were sceptical and cautious that it would be a positive development.

"Devolution is not sorted. What devolution means for us? Does it mean a collaboration, a partnership or a merger? Does it mean a merger with a local organisation or a national organisation? And it's a massive piece of work to get into. So I feel as an organisation a little bit like a rabbit caught in front of the headlight, with devolution being the headlight."

Larger organisations tended to have greater knowledge and understanding than smaller ones, particularly if they had attended meetings and events on the subject, but even they tended to feel that more information was needed before they could fully understand the implications of devolution at an organisation and VCSE sector level.

Outside of the City of Manchester, where the majority of larger organisations and Greater Manchester organisations tend to be based, there was a collective sense amongst focus group participants that devolution would not be a positive development - either in terms of the amount of funding available or the ability to influence policy decisions - and that organisations within the City of Manchester had most to gain. Similarly, it was felt that larger VCSE organisations would be most likely to benefit from devolution as they would have the resources and connections necessary to engage with and influence policy developments.

"I have a lot of concerns about devolution... who owns the partnership? Who are they giving it [money] to? Is anyone from Oldham involved? I don't think so. So I'm quite nervous about the dominance of Manchester; who decides all this? We used to have a good relationship with Oldham council regarding contracts, but who do we go to now? Because the money will not be coming out from them."

\section{Broader relationships with the public sector}

The focus groups also discussed VCSE organisations' broader experiences of working with the public sector. Overall, these discussions were critical of local public sector bodies and the way they worked with VCSE organisations, highlighting a growing sense that VCSEs and the state are becoming increasingly 'disconnected'.

"With the funding being cut, there is a huge emphasis on having to come up with this really huge innovative scheme to get the next grant, when actually what you are doing is really valuable and it's on the nail of the head of what is required in your community. Having to constantly start new things rather than sustaining really good things which already exist, it's pure foolishness."

Common themes across the areas included:

- lack of funding and support for VCSEs

- too much bureaucracy

- limited experience, knowledge and understanding amongst public sector staff of the VCSE sector

- poor communication and consultation 
- poorly designed policies

- unreasonably demanding and overly arduous procurement practices.

However, participants were keen to highlight pockets of good practice within the public sector in its dealings with VCSEs and emphasised that relationships and practices tended to vary over time.

"I think it works when the public sector involves the voluntary sector from the beginning. When you feel that your opinion and input are valued from the start and your involvement from start to finish is considered important."

Key to developing and maintaining effective partnerships with the public sector were the personal relationships VCSEs have with key staff and the trust that is built at an individual level over time.

"It's very much relationships with individuals; the personal relationships that you have developed over the years."

Smaller organisations and social enterprises tended to be more negative than large charities about their relationships with public sector bodies. These VCSE organisations found it particularly challenging to participate in public sector procurement processes with the demanding and bureaucratic processes that were in place.

"It's just got really difficult. Because they went from grants to contracts which is in favour of larger organisations and the council doesn't want to give them in smaller voluntary organisations and it's getting harder and harder to do to application bits." 


\section{Partnership Working: the Private Sector}

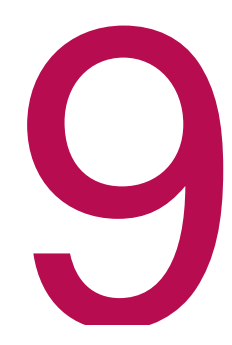

The previous chapter explored respondents' experiences of partnership working with public sector bodies. This chapter moves on to explore their experiences of working with private sector organisations. This area still appears to be new territory for many VCSE organisations. Survey respondents were asked about their direct dealings and experiences of working with private businesses in Greater Manchester.

\section{1. $\quad$ Working with private businesses}

Survey respondents were asked to indicate the extent to which they had direct dealings with private businesses in Greater Manchester. 62 per cent reported that they had some direct dealings, with 21 per cent having a 'great' or 'fair' amount of contact (figure 9.1). In 2012/13, 55 per cent of respondents reported some direct dealings, including 16 per cent having a 'great' or 'fair' amount of contact.

As figure 9.1 shows there was some variation across local area.

Figure 9.1: Extent of direct dealings with private businesses

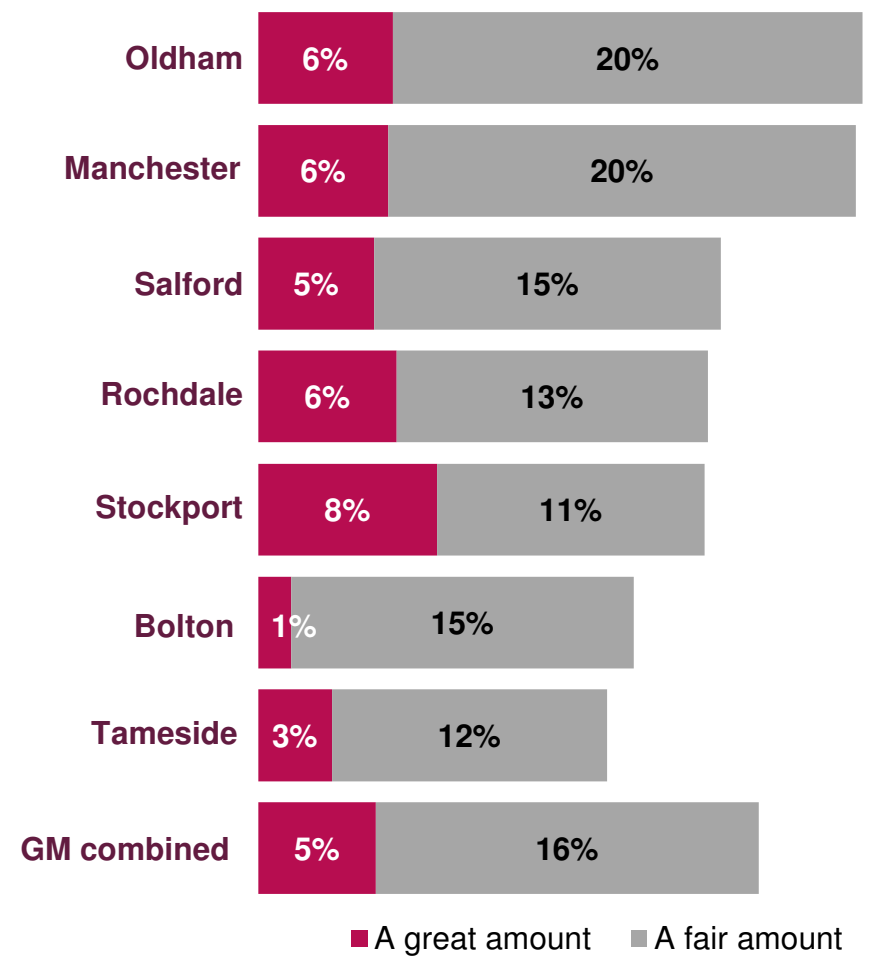

Source: Greater Manchester State of the VCSE sector survey 2016/17 Base: 1,043 
Just two per cent of organisations indicated that they are members of a formal private sector-led consortium, while seven per cent of respondents said their organisation is in formal partnership with any private sector organisations.

Respondents were asked to comment on the influence private businesses have on their organisation's success. Taking all things into account, almost one third (31 per cent) of survey respondents felt that the private business community in Greater Manchester was a positive influence on their organisation's success. This is an increase since the 2012/13 survey when just 19 per cent of survey respondents felt that the private business community in Greater Manchester was a positive influence on their organisation's success.

Figure 9.2: Private business community's influence on VCSE sector organisations' success

Very negative influence

Negative influence

Neither positive nor negative influence

Positive influence

Very positive influence

Don't know
$2 \%$ $3 \%$

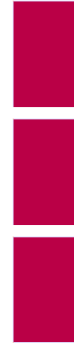

$5 \%$

$11 \%$

Source: Greater Manchester State of the VCSE sector survey 2016/17

Base: 683

\subsection{Qualitative responses on working with private sector businesses}

Focus group participants discussed their views about and experiences of working with private businesses. Overall, VCSE organisations viewed this positively, particularly when it meant forging links with local businesses with strong roots in their area. Aside from donations (which were always welcome) participants identified a range of ways in which they could benefit and learn from partnerships with the private sector:

- approaches to resource management

- organisational development, including strategic and business planning

- recruitment strategies.

"By being a part of the chamber of commerce their events and activities...just the fact of learning how to run an organisation without having the emotional connect, learning to be sustainable, improving in customer service, how to make a strong business plan, strategic growth, recruiting and so on...it has been very important for us." 
However, participants also identified a number of barriers to partnership working with the private sector. These included a 'cultural divide' between the two sectors, and the perception that they have fundamentally different value bases.

"It's a massive culture change though. They don't see costs or anything like we do, they don't like abstract concepts, so it's basically a learning experience for us; a very useful learning experience."

A further barrier to closer working between the two sectors was perceived to be personal relationships and trust that could be developed through effective collaboration.

"It's a huge shift in the culture though, because you have to understand their language and your staff must adapt quickly to that, but if you build some trust with them, we can be very productive."

Participants emphasised how it was easier to develop such relationships when it was clear that certain individuals within private businesses were motivated by a "sincere desire to do good in the community". In addition, some smaller organisations doubted whether they had the necessary skills and confidence to build effective links and ongoing communication with the private sector. 


\section{Partnership Working: Voluntary Community and Social Enterprise Organisations}

The previous two chapters have explored respondents' experiences of working with organisations from the public and private sectors. This chapter discusses survey respondents' views on their work with other VCSE sector organisations.

\subsection{Working with other VCSE organisations}

Survey respondents were asked about the extent to which they had direct dealings with other VCSE sector organisations in both their local area and Greater Manchester as a whole.

The vast majority (90 per cent) had some direct dealings with other VCSE sector organisations in their local area, and as figure 10.1 illustrates, 67 per cent had a 'great' or 'fair amount' of contact. This is similar to the 2012/13 survey, when 88 per cent had some direct dealings with other VCSE organisations in their local area and 60 per cent had a 'great' or 'fair amount' of contact.

As figure 10.1 shows there was some variation across local area. 
Figure 10.1: Extent of direct dealings with VCSE organisations (in local area)

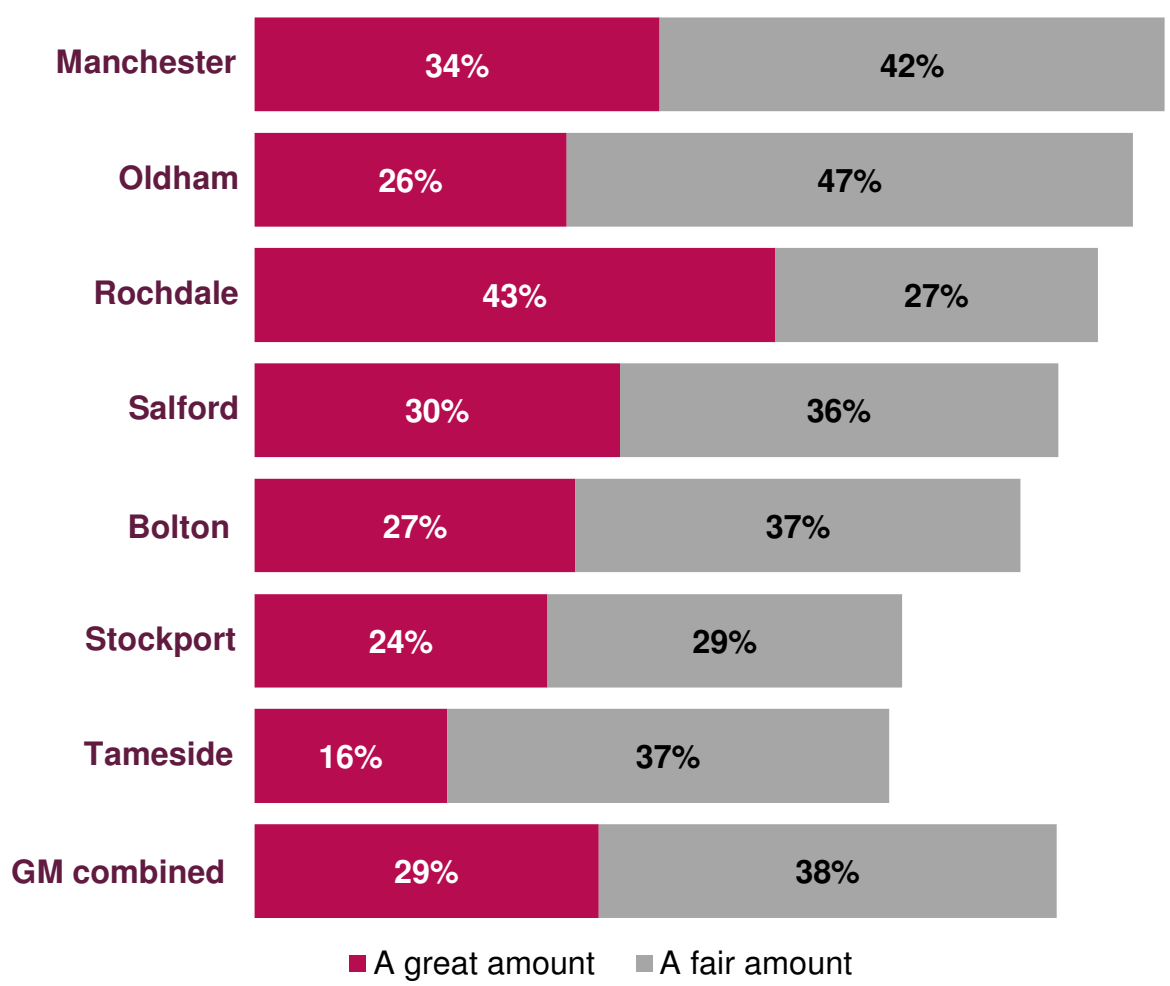

Source: Greater Manchester State of the VCSE sector survey 2016/17

Base: 968

The proportion of respondents reporting they had direct dealings with other VCSE sector organisations in Greater Manchester was lower (70 per cent), along with the proportion who had a 'great' or 'fair amount' of contact (37 per cent). Survey respondents were only asked about their dealings with other VCSE organisations across Greater Manchester in 2016/17.

As figure 10.2 shows there was again some variation across local area. 
Figure 10.2: Extent of direct dealings with VCSE organisations (across Greater Manchester)

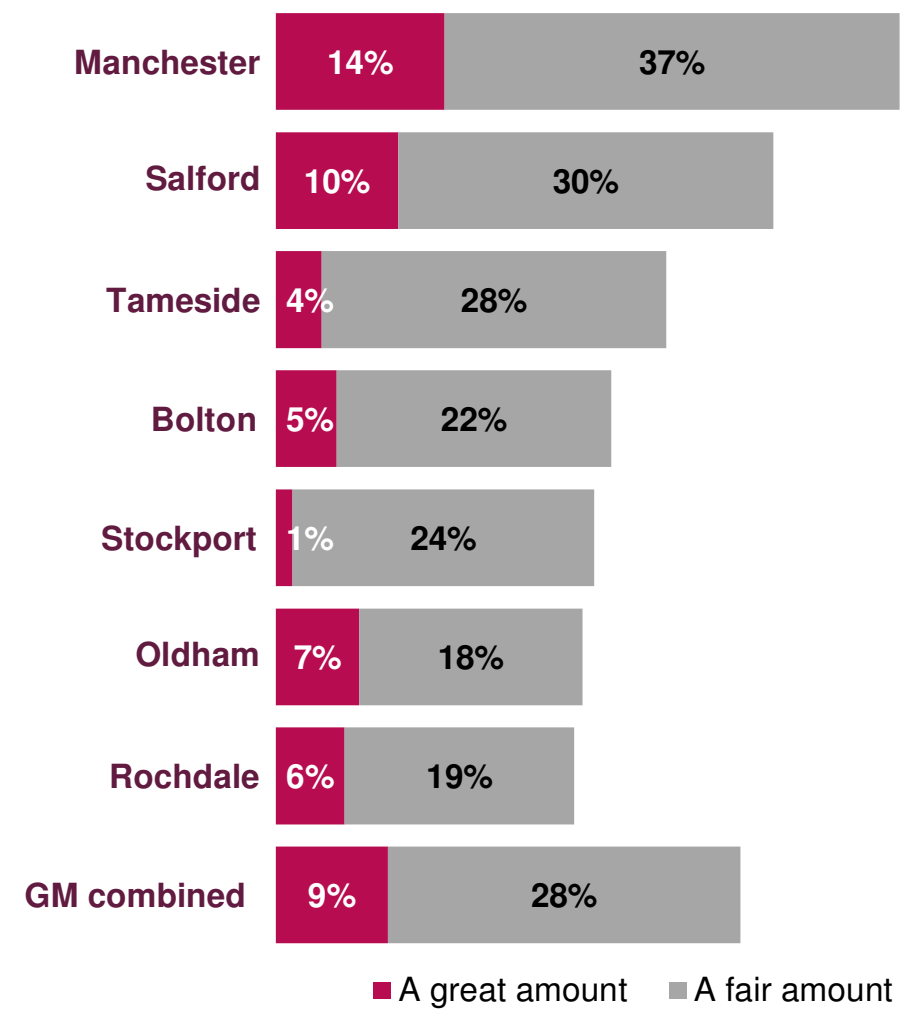

Source: Greater Manchester State of the VCSE sector survey 2016/17

Base: 929

Respondents were asked to reflect on the opportunities they had to work with other VCSE sector organisations in terms of influencing local decisions, delivering local services and networking. Figure 10.3 summarises the responses.

Figure 10.3: Satisfaction with opportunities to work with VCSE organisations

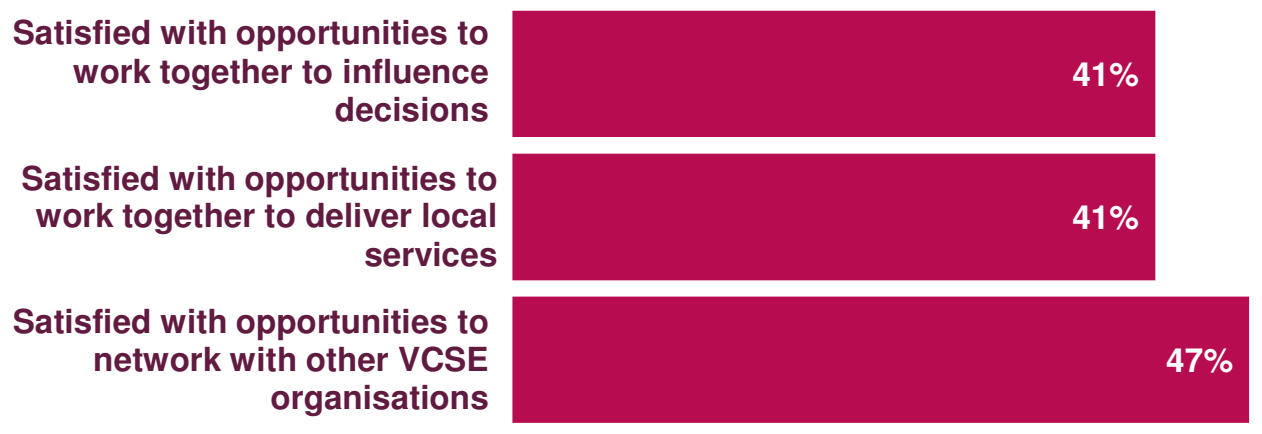

Source: Greater Manchester State of the VCSE sector survey 2016/17

Base: 1,021 (influence decisions) / 1,011 (delivering services) / 1,016 (networking)

This shows that 41 per cent of respondents were satisfied with the availability of opportunities to influence local decisions (48 per cent in 2012/13) and that 41 per cent were satisfied with the availability of opportunities to work together to deliver local services (46 per cent in 2012/13). Almost half (47 per cent) of respondents were also satisfied with opportunities to network with other VCSE organisations. This was a new question added to the latest survey. 
Over one fifth (22 per cent) of respondents said their organisation is a member of a formal VCSE sector consortium while 13 per cent indicated their organisation is in another type of formal partnership with other VCSE organisations to deliver specific services.

\subsection{Qualitative reflections on working with other VCSE organisations}

The focus groups discussed participants' views about and experiences of working in partnership with other VCSE organisations in their area and more widely. Participants were overwhelmingly positive about both their experiences of this type of partnership working and its importance moving forward.

"We work very well with other organisations and the feeling that I get is if I need something, I can ask you; if you need something, feel free to ask me. But beyond that, I want to give something back to the voluntary sector. We can share resources; so when you talk about your DBS, well, we do DBS; so I can do it for you."

Strong personal relationships with key staff in other VCSE organisations, embodied by high levels of trust, were consistently identified as a key factor in enabling effective partnership working.

"If you trust the individual, you trust the organisation; so if you believe that there is something than you can do better by working together, you usually go for it."

"Maybe you've helped them with something in the past and then they reciprocate and that's how these relationships are built. They are the best types of relationships to have with other organisations I think."

Where partnership working had been less effective this was often due to 'mismatches' between organisations. For example, when certain VCSE organisation's capabilities had been misunderstood, where personal relationships had broken down or not properly formed, or where there was a poor 'fit' between organisations values and practices.

"Sometimes it's a fantastic strength if you are similar organisations; if you have the same identity and aspirations. If it turns out you are not, it can get really bad, really quickly. And I guess we all want to protect what we've got, so this you know, suspicion, can be a barrier."

Smaller organisations and social enterprises were particularly positive about the merits of partnership working and many reported pursuing it as a strategy to enable them to 'compete' for funding and contracts. However, there was also caution expressed by some smaller organisations about partnering too closely with some large market-savvy charities which did not appear to share their ethos or values.

"Probably more with similar size and like-minded organisations. Yeah, if you are really like-minded and you do have the same values in terms of the outcomes you want to achieve, certainly. But, I wouldn't work with just anyone in the voluntary sector. And you don't want to be pitted against each other." 


\section{The Future}

This chapter details the responses received to questions about the future in the survey of organisations.

\subsection{Factors assisting or constraining delivery}

Respondents were asked to consider the factors they anticipated assisting or constraining their organisation over the next 12 months. Figure 11.1 illustrates that around half of respondents thought the following factors would assist their organisation over the next year:

- engagement with other VCSE organisations: 50 per cent anticipated this factor assisting their organisation; including 11 per cent who saw this as 'greatly assisting' and 39 per cent 'assisting'

- $\quad$ engagement with public sector bodies: 48 per cent anticipated this factor assisting their organisation; including 12 per cent who saw this as 'greatly assisting' and 37 per cent 'assisting'

- ability to secure other sources of income: 47 per cent anticipated this factor assisting their organisation; including 19 per cent who saw this as 'greatly assisting' and 27 per cent 'assisting'.

In contrast over 30 per cent saw the following factors as constraining their organisation over the next 12 months

- the local economy: 38 per cent anticipated this factor constraining their organisation; including 13 per cent who saw this as 'seriously constraining' and 25 per cent 'constraining'

- ability to recruit volunteers with sufficient skills: 31 per cent anticipated this factor constraining their organisation; including eight per cent who saw this as 'seriously constraining' and 23 per cent 'constraining'. 
Figure 11.1: Factors anticipated as assisting or constraining organisations over the next 12 months

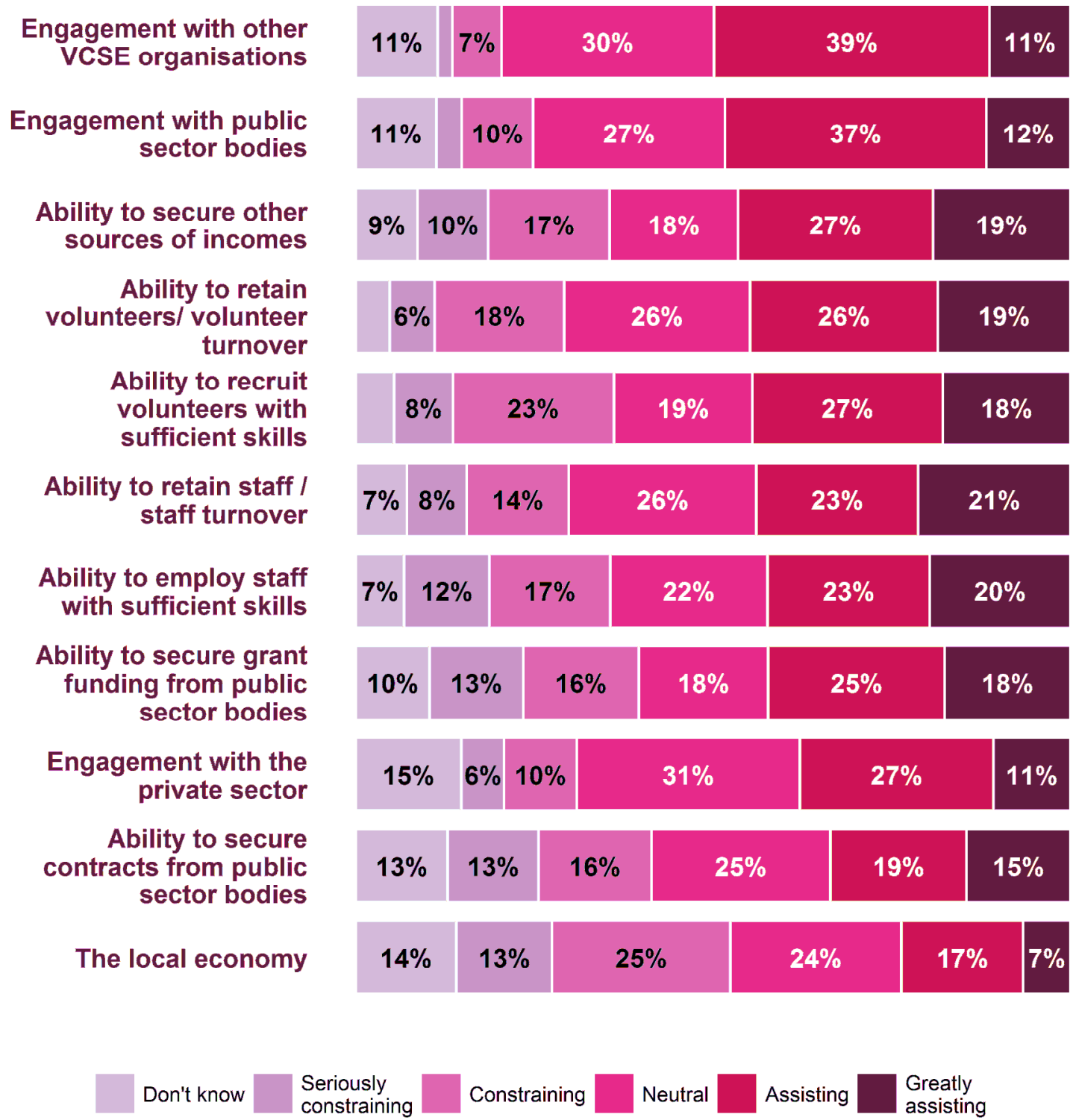

Source: Greater Manchester State of the VCSE sector survey 2016/17

Base: $503-734$

Following on from quantitative questions regarding the factors organisations anticipated assisting or constraining their organisation over the next year, respondents were also asked to provide further qualitative (i.e. written) information about these factors.

Most comments focused on the factors constraining organisations, with issues around accessing funding and recruiting and retaining volunteers and staff with appropriate skills as common themes in the responses:

"With the cuts to local authorities, along with the furthering of austerity policies, I think there is likely to be an increase of pressure on charitable \& voluntary organisations to pick up the slack with little or no additional funding."

"As a small organisation we do not have the resources to focus on income generation from a variety of sources and so any efforts in those areas will simply 
add to workload. The lack of longer term funding prevents a focus on delivery and diverts resources to income generation instead."

"We get volunteers to take up roles on our committee but very few last. It is an increasing problem trying to get people to take on these administrative roles. Getting volunteers to actually join in the community work is not a problem."

"We have difficulty in finding volunteers with the necessary skills"

"Cuts in local authority and NHS funding, Increase in costs due to raise in living wage but not getting sufficient increase in cost from purchasers, difficulty in employing suitable staff due to low wage and high level of responsibility."

There were however some more positive comments received which focused on both existing arrangements and future strategies:

"Partnership work also plays a large part in our future and we are always looking to secure new and long lasting relationships with other VCSE organisations to build upon our skills and experience, share ideas and strengthen our future."

"Networking forum and communicating with businesses."

"With a little effort it is possible to find sources of significant income, e.g. Tesco's Bags of Help."

\subsection{Current and future strategies}

Survey respondents were asked what strategies they are actively pursuing or planning to pursue. Figure 11.2 summarises the responses received and shows that 56 per cent of respondents or more were already doing or planning to do the following

- working more closely with another voluntary/not-for-profit organisation: 64 per cent were already pursuing or planning to pursue this strategy; including 29 per cent who are doing this now and 36 per cent who are planning to do this in the future

- increasing earned income: 60 per cent were already pursuing or planning to pursue this strategy; including 28 per cent who are doing this now and 33 per cent who are planning to do this

- changing the way you run your services or activities: 56 per cent were already pursuing or planning to pursue this strategy; including 22 per cent who are doing this now and 35 per cent who are planning to do this. 
Figure 11.2: Strategies being planned or pursued

Working more closely with another voluntary/not-forprofit organisation

Increasing earned income

Changing the way you run your services or activities

Working more closely with a public sector organisation

Increasing individual donations

Working more closely with a private sector company

Merging with one or more similar organisations

Borrowing money to increase the volume of activity/enter new areas of work

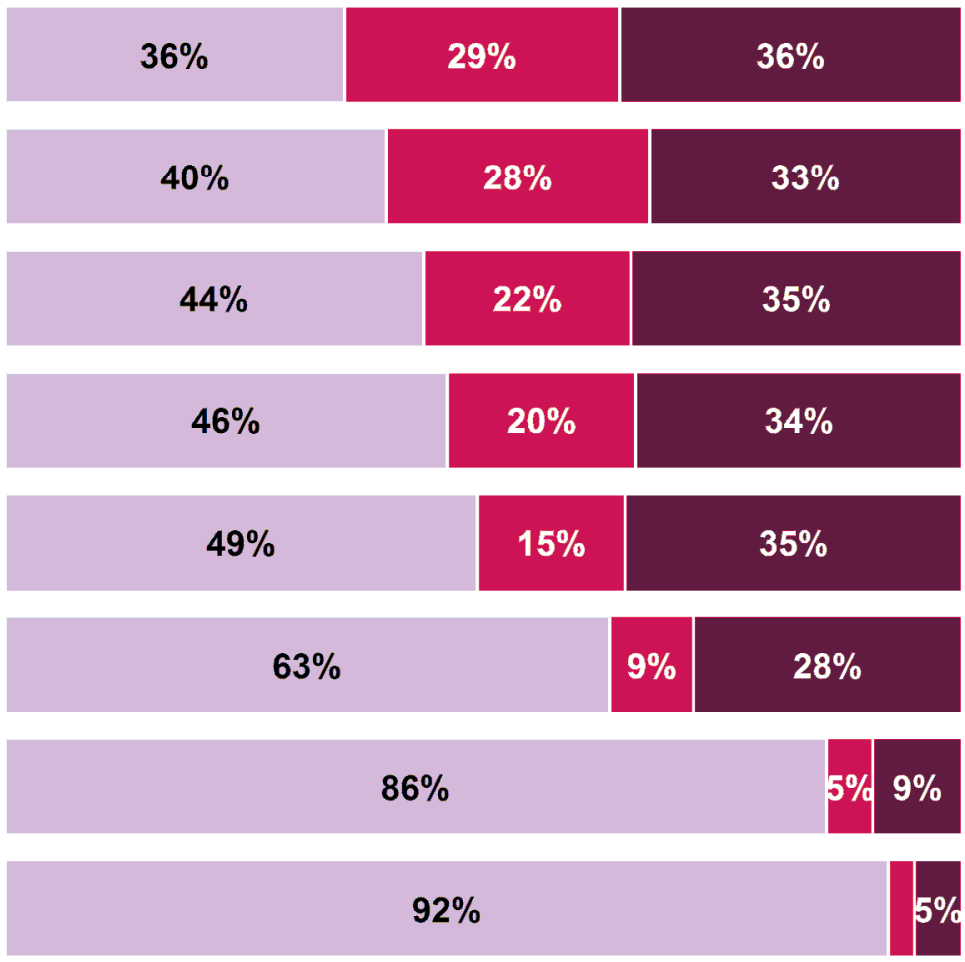

Not doing this now and not planning to do this

Doing this now

Planning to do this

Source: Greater Manchester State of the VCSE sector survey 2016/17

Base: 785-825 


\section{Conclusions}

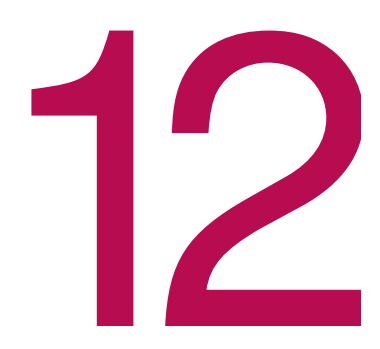

\section{Key findings from the research}

This report provides the main findings of research aimed at improving the understanding of the social and economic impact of the voluntary, community and social enterprise (VCSE) in Greater Manchester.

There are nine key findings from the research:

1. The VCSE sector in Greater Manchester continues to occupy an important strategic position within the city region.

2. The sector in Greater Manchester remains an important economic player, contributing significantly to GVA, but patterns in income, expenditure and the level of reserves suggest that, as in 2013, the sustainability of many organisations may be under threat.

3. The VCSE sector in Greater Manchester continues to provide significant social value.

4. The VCSE sector continues to be a significant employer.

5. Volunteering is an essential and highly valuable feature of the 'identity' of the VCSE sector, however there are challenges associated with volunteering across Greater Manchester.

6. There is a mixed picture in Greater Manchester regarding relationships between the VCSE sector and public sector bodies.

7. Engagement with private businesses has increased and perceptions of the private business sector appear to have improved, although engagement is still relatively low.

8. The VCSE sector in Greater Manchester continues to be well connected internally although most contact appears to be informal.

9. The sector still faces an uncertain future. 


\section{Appendix 1}

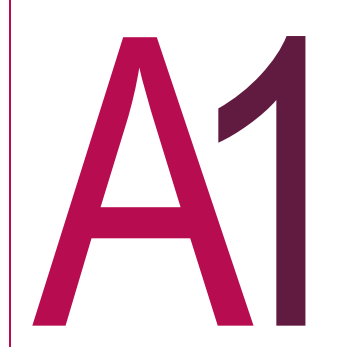

\section{Methodology}

\section{Survey of organisations}

In total 1,166 survey responses were collected overall during September 2016 - January 2017. Responses were received as follows from the ten Greater Manchester local authority areas

- Bolton: 163 responses

- Bury: 18 responses

- Manchester: 201 responses

- Oldham: 116 responses

- Rochdale: 77 responses

- Salford: 198 responses

- Stockport: 88 responses

- Tameside: 140 responses

- Trafford: 29 responses

- Wigan: 55 responses

- Greater Manchester: 81 responses

Responses were collected electronically in a number of ways. One version of a web basedsurvey of organisations was sent out by CRESR to VCSE organisations in six of the individual local authority areas participating in the study.

Responses were received as follows

- Bolton: 62 responses

- Manchester: 63 responses

- Oldham: 65 responses

- Rochdale: 27 responses

- Salford: 54 responses

- Tameside: 65 responses

A further 12 responses were received in Bury where contacts for this area were provided by the local infrastructure organisation in Rochdale. 
In total at least partial responses were received from 348 of the 4,982 organisations that were sent a survey questionnaire: this represents a response rate of seven per cent.

A second web-based version of the survey was also distributed by the local infrastructure organisations to each local authority area participating in the study and additionally Stockport Council in the Stockport local authority area, reaching organisations also included in the original sample and beyond. In addition GMCVO distributed a version of the survey via their networks, also reaching VCSE organisations. The local infrastructure organisations played a key role in boosting the response rate to the survey by utilising their relationships within their areas to encourage organisations to complete a questionnaire.

Responses were received as follows from the versions of the survey distributed more widely by local infrastructure agencies and GMCVO:

- Bolton: 101 responses

- Bury: 6 responses

- Manchester: 138 responses

- Oldham: 51 responses

- Rochdale: 50 responses

- Salford: 144 responses

- Stockport: 88 responses

- Tameside: 75 responses

- Trafford: 29 responses

- Wigan: 55 responses

- Greater Manchester: 81 responses $^{27}$

In total a further 818 responses were collected via this method, giving a higher overall response rate to the survey overall.

The questionnaire was based on the one originally developed for the 'State of the Voluntary Sector Survey' undertaken in Salford in 2010. The questionnaire was revised for the 'Greater Manchester State of the Voluntary Sector' research undertaken in 2012/13 and again for this wave of the survey following input from the Research Steering Group. The Greater Manchester Chief Officers Group also provided additional oversight regarding the survey design and implementation.

The questionnaire provided data on various aspects of the VCSE sector including:

- the scale and scope of its activity, including the roles organisations undertake, the people they support, and the areas they benefit

- the economic impact of its work, including income and expenditure, sources of funding, the role of paid staff and volunteers, and financial sustainability

- relationships with the public sector, including local authorities, public sector health bodies, and a range of other local statutory bodies

\footnotetext{
${ }^{27}$ These responses were collected from organisations who did not consider their work and relationships to be primarily within one Greater Manchester local authority.
} 
- relationships with other local organisations, including VCSE organisations and private businesses.

Where possible the report compares results from the latest survey and the 2012/13 study. Revisions to the questionnaire mean that comparisons are not always possible or appropriate. It is also worth noting that in 2012/13 a large postal survey was the main method of data collection which was supplemented with a web based survey. This is different to the latest study when a web based survey was the primary method of data collection.

When reading the report it is important to acknowledge two key points. First, the results reported are based on the survey responses received. Therefore it is possible that if a different sample of organisations had taken part in the survey different results may have emerged. It is estimated that the results reported are within $+/-2.8$ percentage points of the true value.

Secondly, on a number of occasions the analysis in this report has used extrapolations from the survey responses to provide estimates of totals for all organisations that work in the VCSE sector including:

- the number of clients, users and beneficiaries of the sector

- the total income of the sector

- $\quad$ and the number of FTE paid staff and the number of volunteers and committee/board members that are part of the sector's workforce; including the hours per week that volunteers contribute.

\section{Estimating the number of organisations in the VCSE sector}

Estimating the number of organisations represents a major challenge. This is because a large proportion of organisations are small, local and not formally constituted as charities, limited companies or other recognised forms which require registration (e.g. industrial and provident societies). As a result they do not appear on formal central records such as those held by the Charity Commission or the Department for Business Innovation and Skills (BIS) so are considered 'below the radar' (BTR). Any estimate of the total number of organisations in an area therefore requires information on the numbers of registered and unregistered (i.e. BTR) organisations.

In estimating the total number of organisations in Greater Manchester we drew on information from the following sources:

- The Register of Charities in England and Wales, which indicated 4,279 registered charities with postcodes in Greater Manchester.

- The ratio of charities to non-charities provided in the 'National Survey of Charities and Social Enterprises' (NSCSE), undertaken by Ipsos MORI for Cabinet Office in 2010. This was used to gross the estimate upwards to a total of 5,802 registered organisations, to take account of non-charitable social enterprises.

- Research by NCVO and the University of Southampton ${ }^{28}$ which found that on average there are 3.66 BTR organisations per 1,000 population. If this figure is applied to

\footnotetext{
${ }^{28}$ Mohan, J et al (2010). Beyond 'flat-earth' maps of the third sector: enhancing our understanding of the contribution of 'below-the-radar' organisations. Northern Rock Foundation Briefing Paper
} 
Greater Manchester ${ }^{29}$, it can be estimated that there are 10,088 BTR organisations in the city region. ${ }^{30}$

For the 2013 report, the sampling frame for the NSCSE was used to provide the estimates for the number of formally registered organisations. Unfortunately this survey was subsequently cancelled and has not been repeated due to public sector spending cuts. As such only the ratio of charities to non-charities was taken from this data source, and combined with the number of charities from the charity register.

\section{Estimating the size of organisations in the VCSE sector}

The size of organisations is traditionally measured using their annual income ${ }^{31}$. When the distribution of organisations across Greater Manchester was explored by size category based on income for 2014/15, it showed that the majority of organisations were either micro or small. But the survey was under-representative of BTR organisations (only 33 per cent of survey respondents were identified as BTR), so this did not present an accurate picture of the actual distribution. The figures were therefore adjusted based on the assumption that the estimated 8,006 organisations not included in the survey sample were BTR and micro in size $^{32}$.

Exploring the distribution by size category based on income for 2014/15 also showed some inconsistency with the distribution found in 2013. Therefore, in order to provide the most robust estimate of the distribution of organisations in the VCSE sector by size, data from both waves of the survey have been used to calculate the proportion of organisations estimated to be in each size category.

The outcome of this process is shown in figure 3.1 in Chapter 3 , which demonstrates that 77 per cent of the VCSE sector (an estimated 12,312 organisations) are micro in size, 12 per cent are small (1,983 organisations), eight per cent are medium (1,221 organisations), and two per cent are large (374 organisations).

Introducing the BTR figure produces a much higher estimate for the number and proportion of micro organisations and emphasises the finding that a large proportion of organisations in the VCSE sector in Greater Manchester are very small (90 per cent micro or small). This is similar to the national picture: $\mathrm{NCVO}^{33}$ estimate that 83 per cent of the VCSE sector is made up of micro or small organisations, 14 per cent are medium, and three per cent are large.

\section{Calculating the sector wide totals}

In each case the same three stage method has been used for calculating the sector wide totals:

- $\quad$ stage one: calculate the Greater Manchester averages for each of the four size bands of organisations: 'micro', 'small', 'medium' and 'large': column (a) in table A1

- $\quad$ stage two: multiply the average for each size band (column (a) in table A1) by the estimated number of organisations within that size band (column (b) in table A1) to give the total for each size band of organisations (column (c) in table A1)

\footnotetext{
${ }^{29}$ Based on Office for National Statistics 2015 population estimates

${ }^{30}$ It is important to note that the BTR figure is an estimate based on an average across 46 local authorities. The BTR research found significant variability, with some local authorities reaching over seven BTR organisations per 1,000 population, and in one case exceeding 10.

${ }^{31}$ In exploring organisation size we used the categories developed by NCVO for use in their Almanac series (see e.g. Clark, J et al., 2010)

${ }^{32}$ The basis for these assumptions is discussed in more detail in the methodological annex

${ }^{33}$ UK Civil Society Almanac (2016) NCVO.
} 
- $\quad$ stage three: sum the estimates from stage two (column (c) in table A1) to give a sector wide total estimate (cell (d) in table A1).

This was necessary to take account of noticeable differences in the response rates by organisation size. Failure to do this would lead to upwardly biased estimates: a small number of mainly 'large' organisations create a high mean value that is not representative of the majority of organisations. This is an important point given that we estimate that a large proportion of the sector is made up of 'micro' organisations which tend to have far lower values and not taking into account difference by size of organisations would produce estimates that are much higher.

Table A1: Extrapolations: a worked example (total annual income)

\begin{tabular}{lrrr} 
& $\begin{array}{c}\text { Average income by } \\
\text { size } \\
\text { (a) }\end{array}$ & $\begin{array}{c}\text { Estimated number } \\
\text { of organisations } \\
\text { (b) }\end{array}$ & $\begin{array}{c}\text { Total income } \\
\text { (thousands) } \\
\text { (c) }\end{array}$ \\
\hline Micro (under $£ 10 k$ ) & $£ 2,438$ & 12,312 & $£ 30,012,390$ \\
Small (£10k to $1100 k)$ & $£ 38,844$ & 1,983 & $£ 77,034,137$ \\
Medium (£100k to $11 \mathrm{~m})$ & $£ 320,581$ & 1,221 & $£ 391,502,163$ \\
Large (over $£ 1 \mathrm{~m})$ & $£ 2,201,023$ & 374 & $£ 822,595,202$ \\
\hline Total & & & (b) $£ 1, \mathbf{3 2 1 , 1 4 3 , 8 9 3}$ \\
\hline
\end{tabular}

\section{Focus groups}

To provide a further depth of understanding to some of the themes covered in the State of the Sector Survey, focus groups were undertaken with VCSE organisations in five Greater Manchester districts. The focus groups were held midway through the survey administration and undertaken by the local infrastructure organisation in each area who recruited local organisations to participate in the groups.

A topic guide was devised to help guide discussions and ensure a standardised approach across all local authority areas conducting focus groups. The topic guide was created in partnership between CRESR and the Research Steering Group with CRESR providing advice and guidance on best practice in undertaking this type of research.

The focus groups lasted approximately 1 hour - 1 hour 30 minutes and were digitally recorded where consent was obtained. The recordings were then provided to CRESR who analysed the discussions. Analysis of the discussions is included in the relevant chapters of this report.

The topics discussed in the focus groups concentrated on four key themes: volunteering, working with the public sector, working with other VCSE organisations and working with the private business sector.

Focus groups in each district took place with VCSE organisations from that area with participants selected by organisation type.

The following groups took place in each area with the following organisations:

- Bolton - two groups (one with small VCSE groups and one with registered charities)

- Manchester - two groups (one with small VCSE groups/social enterprises and one with registered charities) 
- Oldham - one group (with small VCSE groups/registered charities)

- Salford - three groups (with participants selected by organisation type specifically registered charities, small VCSE groups and social enterprises )

- $\quad$ Tameside - one group (with small VCSE groups/registered charities).

\section{Legal status of responding organisations}

Respondents to the questionnaire were asked to identify the legal status of their organisation. For this question it was possible for organisations to select registered charity in addition to identifying their legal form. Figure A1 below shows that 30 per cent were a group with a constitution, but not registered charities and 28 per cent of organisations were a company limited by guarantee and that separate to identifying their legal status almost half of respondents, 49 per cent, identified that their organisation was a registered charity.

These results are similar to those in the $2012 / 13$ survey when:

- 38 per cent of organisations were a group with a constitution, but not registered charities

- 22 per cent were companies limited by guarantee (26 per cent 2010)

- four per cent of organisations had no legally constituted form

- 48 per cent of respondents identified that their organisation was a registered charity.

Figure A1: The legal status of responding organisations

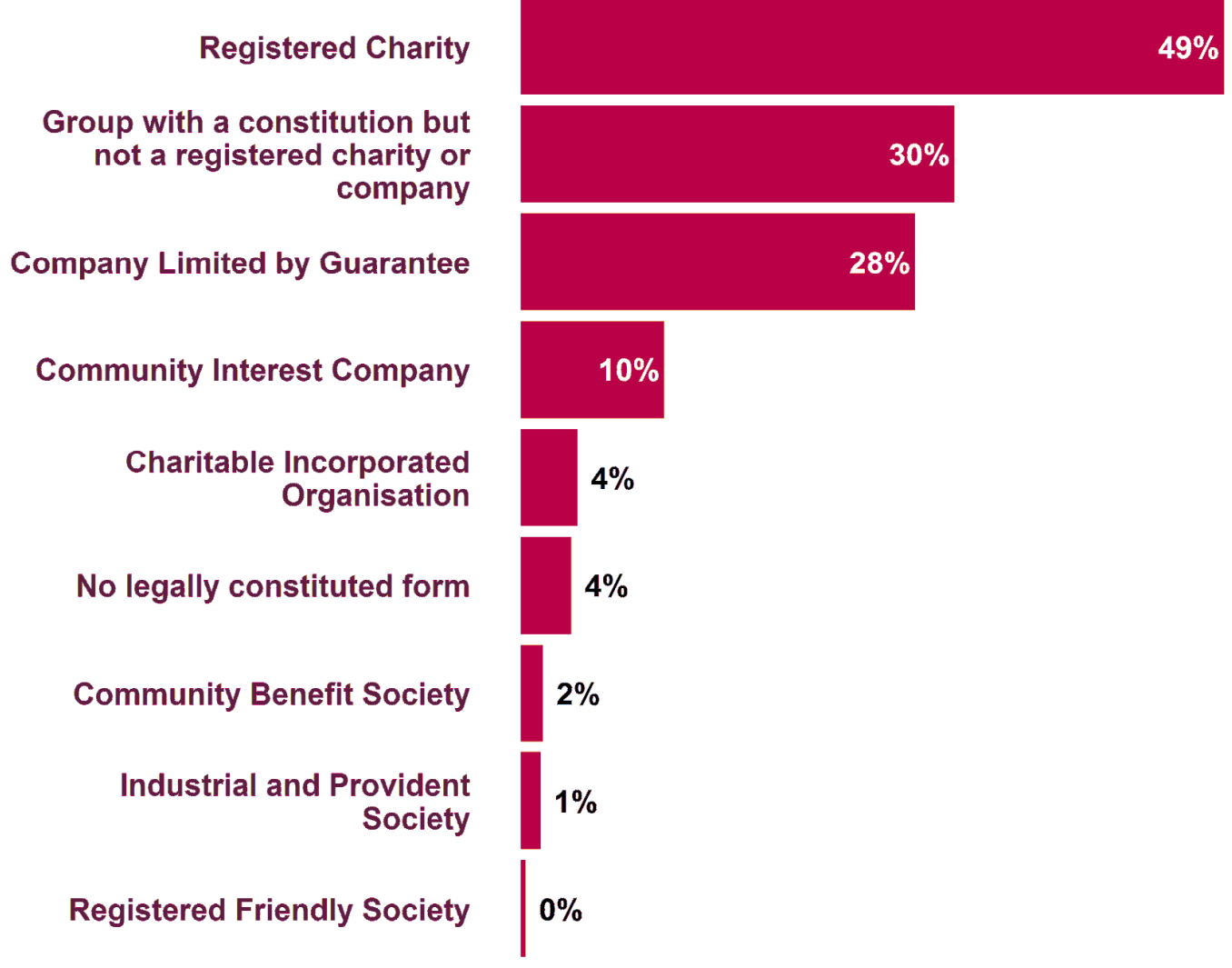

Source: Greater Manchester State of the VCSE sector survey 2016/17 Base: 1,151 
Since the earlier study there has been a change in the legal forms available to VCSE organisations and this question was subsequently revised for the latest study. The changes above are likely to reflect these new opportunities. Charitable Incorporated Organisation and Community Benefit Society were additional options included in the latest version of the questionnaire. 


\section{Sheffield Hallam University}

Greater Manchester state of the voluntary, community and social enterprise sector 2017 : A report on social and economic impact

DAMM, Christopher <http://orcid.org/0000-0002-7355-3496>, PRINOS, Ioannis and SANDERSON, Elizabeth <http://orcid.org/0000-0003-1423-1670>

Available from the Sheffield Hallam University Research Archive (SHURA) at:

http://shura.shu.ac.uk/16667/

\section{Copyright and re-use policy}

Please visit http://shura.shu.ac.uk/16667/ and http://shura.shu.ac.uk/information.html for further details about copyright and re-use permissions. 